\title{
Discriminative Power of Typing Features on Desktops, Tablets, and Phones for User Identification
}

\author{
AMITH K. BELMAN and VIR V. PHOHA, Syracuse University, USA
}

\begin{abstract}
Research in Keystroke-Dynamics (KD) has customarily focused on temporal features without considering context to generate user templates that are used in authentication. Additionally, work on KD in hand-held devices such as smart-phones and tablets have shown that these features alone do not perform satisfactorily for authentication. In this work, we analyze the discriminatory power of the most-used conventional features found in the literature, propose a set of context-sensitive or word-specific features, and analyze the discriminatory power of proposed features using their classification results. To perform these tasks, we use the keystroke data consisting of over $650 \mathrm{~K}$ keystrokes, collected from 20 unique users during different activities on desktops, tablets, and phones, over a span of two months. On an average, each user made $12.5 \mathrm{~K}, 9 \mathrm{~K}$, and $10 \mathrm{~K}$ keystrokes on desktop, tablet, and phone, respectively.

We find that the conventional features are not highly discriminatory on desktops and are only marginally better on hand-held devices for user identification. By using information of the context, a subset (derived after analysis) of our proposed word-specific features offers superior discrimination among users on all devices. We find that a majority of the classifiers, built using these features, perform user identification well with accuracies in the range of $90 \%$ to $97 \%$, average precision and recall values of 0.914 and 0.901 , respectively, on balanced test samples in 10 -fold cross validation. We also find that proposed features work best on handheld devices. This work calls for a shift from using conventional KD features to a set of context-sensitive or word-specific KD features that take advantage of known information such as context.
\end{abstract}

CCS Concepts: • Security and privacy $\rightarrow$ Biometrics; $\bullet$ Human-centered computing $\rightarrow$ Heuristic evaluations; Text input;

Additional Key Words and Phrases: Keystroke dynamics, context-sensitive features, discriminative power, typing, desktop, phone, tablet

ACM Reference format:

Amith K. Belman and Vir V. Phoha. 2020. Discriminative Power of Typing Features on Desktops, Tablets, and Phones for User Identification. ACM Trans. Priv. Secur. 23, 1, Article 4 (February 2020), 36 pages.

https://doi.org/10.1145/3377404

\section{INTRODUCTION}

The rise in the popularity of biometrics stems from its inherent property that eliminates the need for a person to remember and reproduce complex secretive information to authenticate oneself. Possession of such secretive information, such as Personal Identification Number (PIN) and Password, is not only risking its theft but also risking forgetting it; either case leads to unnecessary

This work was supported in part by the National Science Foundation (NSF) under Grant SaTC-1527795.

Authors' addresses: Amith K. Belman and V. V. Phoha, Syracuse University, Department of Electrical Engineering and Computer Science, Syracuse, NY, 13244, USA; emails: \{akamathb, vvphoha\}@syr.edu.

Permission to make digital or hard copies of all or part of this work for personal or classroom use is granted without fee provided that copies are not made or distributed for profit or commercial advantage and that copies bear this notice and the full citation on the first page. Copyrights for components of this work owned by others than ACM must be honored. Abstracting with credit is permitted. To copy otherwise, or republish, to post on servers or to redistribute to lists, requires prior specific permission and/or a fee. Request permissions from permissions@acm.org.

(C) 2020 Association for Computing Machinery.

2471-2566/2020/02-ART4 \$15.00

https://doi.org/10.1145/3377404

ACM Transactions on Privacy and Security, Vol. 23, No. 1, Article 4. Publication date: February 2020. 
complications regarding an individual's identity. Biometrics focuses on authenticating a person based on "who they are" rather than "what they know," which is a prime reason for its growth in popularity and research (e.g., see Delac and Grgic [17] and Blasco et al. [9]). Behavioral biometrics is a branch of biometrics that focuses on using a person's behavior or way of doing a task as means of authentication. These tasks can be any common, day-to-day tasks such as walking, sleeping, talking, typing, and so on. As interactions with computers and other smart devices such as phones and tablets have become an essential part of modern life, a person's style of interaction with them can be used as a powerful means of behavioral biometrics. Typing is a common form of interaction, where a person provides input for these devices either on keyboards or touch screens, thus making research in Keystroke Dynamics (KD) popular. Research in KD has grown far and wide, Umphress and Williams [56], in their work, demonstrated that keystroke behavior on keyboards/typewriters was indeed a distinguishable trait among users while more recent research has shown that $\mathrm{KD}$ can also be used on other devices that involve typing, such as phones and tablets $[14,37]$. A considerable amount of research has also explored the effects of the type of text used for KD-that is, fixed text vs free text [1]. The problem of authenticating users by their typing behavior has also been addressed from multiple perspectives as far as the underlying algorithms are concerned. Fuzzy logic [29], Neural Networks [4], mini-batch bagging [22], and pairwise user coupling [34] techniques have been explored in an attempt to improve accuracies and complexity of KD systems. New approaches that use special hardware [50,57] for KD systems are also a promising avenue for researchers. One such work from Sulong et al. [51] explored new features such as a combination of maximum pressure exerted on the keyboard and time latency between keystrokes and showed their proposed system was an effective biometric-based security system.

In recent years, KD has been used in a myriad of applications, such as continuous authentication [35], gender detection [54], age detection [39], fatigue detection [55], mood disturbance detection [59], and lie detection [33], to name a few. There have also been numerous attempts on side-channel attacks on keystrokes based on the acoustic emanations that occur when a person types on physical keyboards. Asonov and Agrawal [6] trained neural networks to recognize the key pressed using the sounds emanated by their press. In their work, they used FFT on $2 \mathrm{~ms}$ windows sound recordings and tested with recordings from varying distance from 1 meter to 15 meters. A similar work carried out by Zhu et al. [58] explores attacks using the acoustic emanations assuming that the different keys pressed are not contextually related. Using the Time Difference of Arrival (TDoA) method, they were able to recover about $72 \%$ of the keystrokes. With a modification of TDoA approach with mm-level audio ranging on a single phone, Liu et al. [30] were able to recover $94 \%$ of keystrokes in their experiments. Although research in KD has been advancing rapidly, there have been very few attempts to understand the impact of context on the features that are used for KD and even the few attempts made were not exhaustive enough.

\subsection{Contributions}

The key contributions of our research work detailed in this article can be summarized as follows:

1.1.1 Analysis of Discriminatory Power of Conventional Keystroke Features for User Identification across Three Most Common Devices. We present our findings, from analysis of conventional KD features, on the three most commonly used devices: desktop, laptop, and phone. We find that conventional features do not separate and hence identify a user's keystroke data efficiently and are simplistic in disregarding the context of these features.

1.1.2 Propose, Analyze, and Evaluate a New Set of Context-sensitive Features across Devices. We propose a set of context-sensitive or word-specific features after analyzing the difference in the discriminative capacity of these features in contrast to the conventional features. We find that 
context-sensitive features are better for user identification on all three devices. The results of user identification show competitive accuracies using proposed features.

1.1.3 Tasks Performed During Data Collection Approximate Real-life Scenarios. To make our study as encompassing as possible for $\mathrm{KD}$, we ensure that the tasks performed by participants, in our data collection efforts, are very close to real-life scenarios. We make sure to include tasks such as transcription for fixed text and browsing and Q \& A for free text, which simulate most of the situations that users encounter.

1.1.4 Provide Insights into Efficacy of Features for Continuous Authentication on Different Device Categories. We also provide mathematical justification of the performance improvements in user identification using proposed features.

KD on desktops, tablets, and phones, which are the three most popular types of devices that people use every day, are all analyzed and reported. This work will help gain a deeper insight into what features might work better for continuous authentication on different device categories. Some initial applications are in the field of continuous authentication of user or as a second factor authentication in existing systems with typing as one of the interfaces. Scenarios such as online examinations, competitions, and remote work environments might benefit from the insights provided by our work. Situations where context is known can be handled much more accurately with the help of the proposed features described here. This article is also aimed at inspiring exploration of novel features that use additional information such as the language and contexts of features to their advantage.

\section{RELATED WORK}

Researchers of KD have explored and studied the effectiveness of various keystroke features for a long time now $[40,45,53]$. In numerous research, available in already existing literature, it is clear that $\mathrm{KD}$ is a promising dimension for authentication and verification $[3,13,25,27,36,43,44$, 46]. Initial work of Obaidat and Sadoun [38], on the analysis of features for verification of users based on keystroke dynamics, is a continuation of three previously published papers by the authors. They demonstrated the advantages of using both KeyHold value and flight time, as opposed to just one of them. They showed that there was a significant dip in misclassification errors when the features were used in combination. The study consisted of 225 samples collected each day, for eight days, from 15 users. Each sample was seven characters in length on average. The authors claimed $100 \%$ accuracy on this set of users using fuzzy ARTMAP, Radial Basis Function Network, and learning vector Quantization. The main drawbacks of this study were the limited number of users, the small text set of seven characters each, and confining the data to only one device that is the desktop. Huang et al. [24] made one of the most recent efforts to analyze the effects of text filtering on keystroke biometrics. The authors have used the work of Gunetti and Picardi [20] as their baseline to present their case on the effect of text filtering. It is worth noting that this study concluded that nearly $23.3 \%$ of all free-text keystrokes were gibberish. Gibberish was defined by the authors as text belonging to four main categories: Repetitive, Gaming ("a," "s," "d," and "w"), Distinct (too few distinct characters) and Lengthy (long strings with length more than 20). It is also shown that the density curves of many Di-graphs changed drastically after filtering. The authors' concluding remark is that filtering of gibberish has no effect on FAR but significantly improves FRR. Using two main filtering techniques, Regular Expressions and spell checkers, this study establishes that the context from which a feature is extracted plays an important role in the performance of the keystroke-based system. Balagani et al. [8] analyzed the discriminability of heterogeneous and aggregate feature vectors with different combinations of keystroke features. The authors used ReliefF, correlation-based feature selection, and consistency-based feature selection 
to perform feature selection analysis. This work provided theoretical proof backed by empirical analysis to confirm that heterogeneous feature vectors were more discriminative than aggregate feature vectors.

Alsultan et al. [5] explored non-conventional keystroke features. "Semi-Timing" and "Editing" features (as the authors call them) and their advantages were analyzed in this work. Semi-timing features defined were the Words Per Minute (WPM), Negative Up-Down (press second key before releasing first) and Negative Up-Up (release second key before releasing first). The Editing features defined regarded the general tendencies and mannerisms of a user, such as Error rate (number of backspaces), Caps-lock Usage, and Shift Usage (resealed before/after letter). The use of Ant Colony Optimization (ACO) feature reduction, leading to five features that contributed the most (Negative Up-Down, Error Rate, Right-Shift-Before, Left-Shift-After, Left-Shift-Before) is a valuable insight. Decision trees and Support Vector Machines were used for a comparative study on classifier performance; Decision Trees had a slightly better performance, possibly due to the inbuilt feature reduction property of Decision Trees. The study presents competitive False Acceptance Rates (FAR) and False Reject Rates (FRR), 0.011 and 0.26, respectively, on a dataset of 30 users. Numerous researchers (Azevedo et al. [7], Davoudi and Kabir [16]) have also explored different feature selection techniques, distance measures, and effects of different languages [21] on a KD system. Sun et al. [52], in their work, described a group of secondary features such as shift and comma that had been previously overlooked as noise and also explored their effectiveness for user classification.

\section{RELATED CONTEXT-BASED WORK}

The effects of linguistic context on KD was explored by Goodkind et al. [19]. In their work, authors raised several important questions regarding the treatment of keystroke features, with respect to word boundaries and part-of-speech. Another research that comes close to our work was carried out by Sim and Janakiraman [48], in which the authors showed that features extracted from DiGraphs and Tri-Graphs were not discriminative enough in free text. The authors rightly suggest that embedding of these features plays a role in their effectiveness. In both, authors fall short of proposing any word-based features and also limit their studies to desktops only.

We show with empirical analysis that conventional features for $\mathrm{KD}$ are inadequate and can be greatly improved by factoring in knowledge of the language being typed. We propose a set of context-based features and draw mathematical insights for their better performance. We perform our analysis on the data collected from three of the most common devices that the current populace interacts with: the desktop, the tablet, and the phone. From our literature survey, we also note that the search for optimal features for $\mathrm{KD}$ has not been exhausted, and the research community is actively pursuing analysis of non-conventional feature extraction. This is one of the incentives for us to propose our word-specific features.

\section{CONVENTIONAL FEATURES}

We consider all the temporal features that are the building units of conventional features for KD (see Teh et al. [53]). All research on KD has the logging of keystrokes in common. When a user types, a log of each key pressed and released is stored along with the timestamps of these events, from which a host of features are extracted. A Uni-graph is any single key being pressed and released. Similarly, a Di-graph is any two consecutive keys being pressed and released. Uni-graphs and Di-graphs are the basic entities of $\mathrm{KD}$ and can be used to understand the temporal features. For example, if a user types "the," then "t," "h," and "e" are the Uni-graphs and "th" and "he" are the Di-graphs. For any Di-graph formed by keys $K_{i}$ and $K_{i+1}$, the following temporal features can be extracted: 


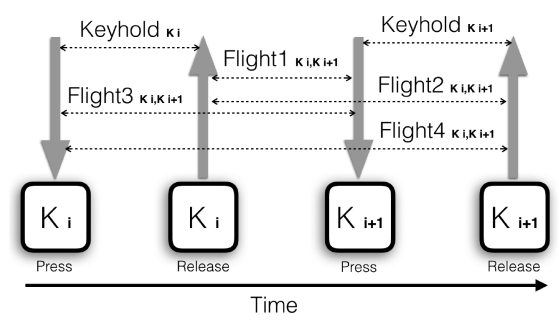

Fig. 1. Features extracted from the temporal data of keys $K_{i}$ and $K_{i+1}$.

(a) KeyHold $K_{i}: K_{i}$ Release $-K_{i}$ Press,

(b) KeyHold $K_{i+1}: K_{i+1}$ Release $-K_{i+1}$ Press,

(c) Flight $1_{K_{i} K_{i+1}}: K_{i+1}$ Press $-K_{i}$ Release,

(d) Flight $2_{K_{i} K_{i+1}}: K_{i+1}$ Release - $K_{i}$ Release,

(e) Flight $3_{K_{i} K_{i+1}}: K_{i+1}$ Press $-K_{i}$ Press,

(f) Flight $4_{K_{i} K_{i+1}}: K_{i+1}$ Release $-K_{i}$ Press,

where $K_{i}$ Press and $K_{i}$ Release correspond to the time when $K_{i}$ was pressed and released, respectively, and so on. Features (a) and (b) are from Uni-graphs, (c)-(f) are from Di-graphs. We can also observe that the values of Flight 1 and Flight 2 could be negative, as it is common for users to press and release a second key before the release of the first key. Figure 1 illustrates these temporal features that are integral to the discussions throughout this article.

Conventional KD research uses features that are formed out of these basic temporal features. Each feature is generally sorted into separate groups based on characters that they are derived from; for example, all KeyHold values for character "a" are grouped separately from those of character "b," and so on. Descriptive features are then extracted from these groups and stored as information for the user's template. The groups can be Uni-graphs, Di-graphs, Tri-graphs, and so on. The features extracted and studied in this manner are referred to as "Conventional Features" in the following sections. Table 1 describes the conventional features that are widely used in literature. Conventional features, as shown in Table 1, include KeyHold, Flight1, Flight2, Flight3, and Flight4. While KeyHold is extracted from a Uni-graph, the rest are extracted from Di-graphs. A brief description for each feature is also provided in the table.

Table 2 demonstrates the extraction of these features with the help of an example string "this is that." The example string can be indexed as $t_{1} h_{1} i_{1} s_{1}{ }_{1} i_{2} s_{2}{ }_{2} t_{2} h_{2} a_{1} t_{3}$ (characters indexed per occurrence,_represents "space”). The conventional Uni-graph and Di-graph features extracted from the example string are simplistic in grouping together the values of Uni-graphs and Di-graphs disregarding the context of their occurrence. For example, KeyHold values of Uni-graph " $\mathrm{t}$ " are grouped. Conventional features do not distinguish where the values occur and most descriptive features derived from them will be aggregating values from entire pieces of text to be stored as templates for a user's typing behavior.

\section{PROPOSED CONTEXT-SENSITIVE FEATURES}

In our approach, we focus on extracting descriptive features from words. For the purpose of simplicity, we limit our language to English, therefore all characters and words considered in this research are in English. We define words in the domain of $\mathrm{KD}$ as a set of consecutive keystrokes, preceded and succeeded by "space" or punctuation ("shift" is generally used for most punctuation) that form an English word. For example, if a user intends to type "the" but after typing "th" presses "a" by mistake and uses "backspace" to rectify this mistake, the sequence of keystrokes would be 
Table 1. Conventional Features Extracted from Uni-Graphs and Di-Graphs with Their Brief Description

\begin{tabular}{|c|c|}
\hline Feature & Description \\
\hline \hline KeyHold & $\begin{array}{c}\text { Time duration for which a key } \\
\text { is held down. }\end{array}$ \\
\hline Flight1 & $\begin{array}{l}\text { Time between release of first } \\
\text { key and press of second key. }\end{array}$ \\
\hline Flight2 & $\begin{array}{l}\text { Time between release of first } \\
\text { key and release of second key. }\end{array}$ \\
\hline Flight3 & $\begin{array}{l}\text { Time between press of first } \\
\text { key and press of second key. } \\
\text { Fime between press of first } \\
\text { key and release of second key. }\end{array}$ \\
\hline \multicolumn{2}{|c}{} \\
\hline
\end{tabular}

Table 2. Conventional Features Extracted from an Example String "this is that"

\begin{tabular}{|c|c|}
\hline$U$ & KeyHold \\
\hline $\mathrm{t}$ & $\left(t_{1 R^{-}}-t_{1 P}\right),\left(t_{2 R}-t_{2 P}\right),\left(t_{3 R}-t_{3 P}\right)$ \\
\hline $\mathrm{h}$ & $\left(h_{1 R}-h_{1 P}\right),\left(h_{2 R}-h_{2 P}\right)$ \\
\hline $\mathrm{i}$ & $\left(i_{1 R}-i_{1 P}\right),\left(i_{2 R}-i_{2 P}\right)$ \\
\hline$S$ & $\left(s_{1 R}-s_{1 P}\right),\left(s_{2 R}-s_{2 P}\right)$ \\
\hline- & $\left(-1 R^{-} \_1 P\right),\left({ }_{2} 2 R^{-} \_2 P\right)$ \\
\hline$a$ & $\left(a_{1 R}-a_{1 P}\right)$ \\
\hline
\end{tabular}

\begin{tabular}{|c|c|c|c|c|}
\hline$D$ & Flight1 & Flight2 & Flight3 & Flight4 \\
\hline th & $\begin{array}{c}\left(h_{1 P}-t_{1 R}\right), \\
\left(h_{2 P}-t_{2 R}\right)\end{array}$ & $\begin{array}{c}\left(h_{1 R}-t_{1 R}\right) \\
\left(h_{2 R}-t_{2 R}\right)\end{array}$ & $\begin{array}{c}\left(h_{1 P}-t_{1 P}\right) \\
\left(h_{2 P}-t_{2 P}\right)\end{array}$ & $\begin{array}{l}\left(h_{1 R}-t_{1 P}\right) \\
\left(h_{2 R}-t_{2 P}\right)\end{array}$ \\
\hline hi & $\left(i_{1 P}-h_{1 R}\right)$ & $\left(i_{1 R}-h_{1 R}\right)$ & $\left(i_{1 P}-h_{1 P}\right)$ & $\left(i_{1 R}-h_{1 P}\right)$ \\
\hline is & $\begin{array}{l}\left(s_{1 P}-i_{1 R}\right), \\
\left(s_{2 P}-i_{2 R}\right)\end{array}$ & $\begin{array}{l}\left(s_{1 R}-i_{1 R}\right), \\
\left(s_{2 R}-i_{2 R}\right) \\
\end{array}$ & $\begin{array}{l}\left(s_{1 P}-i_{1 P}\right), \\
\left(s_{2 P}-i_{2 P}\right)\end{array}$ & $\begin{array}{l}\left(s_{1 R}-i_{1 P}\right) \\
\left(s_{2 R}-i_{2 P}\right)\end{array}$ \\
\hline $\mathrm{s}_{-}$ & $\begin{array}{l}\left(-1 P^{\left.-s_{1 R}\right)},\right. \\
\left(-2 P^{\left.-s_{2 R}\right)}\right.\end{array}$ & $\begin{array}{l}\left(-1 R^{-} S_{1 R}\right), \\
\left(-2 R^{-} S_{2 R}\right)\end{array}$ & $\begin{array}{l}\left(-1 P^{\left.-s_{1 P}\right)},\right. \\
\left({ }_{2} P^{-s_{2 P}}\right)\end{array}$ & $\begin{array}{l}\left(-1 R^{-S_{1 P}}\right) \\
\left({ }_{2} R^{-} s_{2 P}\right)\end{array}$ \\
\hline$\_$i & $\left(i_{2 P^{-}}-1 R\right)$ & $\left(i_{2 R^{-}-1 R}\right)$ & $\left(i_{2 P^{-}}-1 P\right)$ & $\left(i_{2 R^{-} \_1 P}\right)$ \\
\hline t & $\left(t_{2 P^{-}-2 R}\right)$ & $\left(t_{2 R^{-}-2 R}\right)$ & $\left(t_{2 P^{-}-2 P}\right)$ & $\left(t_{2 R^{-}-2 P}\right)$ \\
\hline ha & $\left(a_{1 P}-h_{2 R}\right)$ & $\left(a_{1 R}-h_{2 R}\right)$ & $\left(a_{1 P}-h_{2 P}\right)$ & $\left(a_{1 R^{-}} h_{2 P}\right)$ \\
\hline at & $\left(t_{3 P}-a_{1 R}\right)$ & $\left(t_{3 R}-a_{1 R}\right)$ & $\left(t_{3 P}-a_{1 P}\right)$ & $\left(t_{3 R}-a_{1 P}\right)$ \\
\hline
\end{tabular}

$U$ : Uni-Graph, $D$ : Di-Graph. $t_{1 R}$ stands for release of key $t_{1}$ (the subscript 1 stands for the first occurrence of " $\mathrm{t}$ ") and $t_{1 P}$ stands for press of key $t_{1}$ and so on.

"t"+"h"+"a"+"backspace"+"e." Though the text on screen reads "the," the actual keystrokes performed were different; hence, we do not consider this the typing of a word. We use this concept whenever we refer to a "word" throughout this article.

Proposed features are shown in Table 3, extracted for each occurrence of a word as opposed to Uni-graphs or Di-graphs and are composed of "WordHold": the time taken to type an entire word (from first press to last release); "AvgKeyHold": the average of all KeyHold values in the word; "AvgFlight1," "AvgFlight2," "AvgFlight3," and "AvgFlight4": the average of the respective Flight values for Di-graphs in the word; "StdKeyHold": the standard deviation of all KeyHold values in the word; "StdFlight1," "StdFlight2," "StdFlight3," and "StdFlight4": the standard deviation of the respective Flight values for Di-graphs in the word. At this point in the discussion, we have merely proposed these features and plan to investigate the efficiency of these proposed and conventional features as the discussion proceeds. Table 4 demonstrates the extraction of these features with the help of the same example string considered in Table 2: "this is that." The example string can be indexed as $t_{1} h_{1} i_{1} s_{1}{ }_{1} i_{2} s_{2}{ }_{2} t_{2} h_{2} a_{1} t_{3}$ (characters indexed per occurrence, _represents "space"). With our proposed set of features, we take advantage of the context by localizing the feature extraction to words in the given text. From each occurrence of a word, as shown in Table 4, the proposed features do not group based on Uni-graphs and Di-graphs but rather based on words they appear in. The key takeaways from this section are: Conventional features do not distinguish where the values occur; and most descriptive features derived from them will be aggregating values from entire pieces of text. Proposed features factor in the effect of context by aggregating feature values only within the range of a word.

In following sections, we explain how conventional features are not ideal and consider the rich information that lies in context of these features. We present thorough analysis of the 
Table 3. Proposed

Context-sensitive Features and Their Brief Description

\begin{tabular}{|c|c|}
\hline Feature & Description \\
\hline \hline WordHold & $\begin{array}{c}\text { Time between the } \\
\text { press of first key and } \\
\text { the release of last key } \\
\text { in the word. }\end{array}$ \\
\hline AvgKeyHold & $\begin{array}{c}\text { Average KeyHold } \\
\text { values within a word. }\end{array}$ \\
\hline AvgFlight1 & $\begin{array}{c}\text { Average Flight1 } \\
\text { values within a word. }\end{array}$ \\
\hline AvgFlight2 & $\begin{array}{c}\text { Average Flight2 } \\
\text { values within a word. }\end{array}$ \\
\hline AvgFlight3 & $\begin{array}{c}\text { Average Flight3 } \\
\text { values within a word. }\end{array}$ \\
\hline AvgFlight4 & $\begin{array}{c}\text { Average Flight4 } \\
\text { values within a word. }\end{array}$ \\
\hline StdKeyHold & $\begin{array}{l}\text { Standard deviation of } \\
\text { KeyHold in a word. }\end{array}$ \\
\hline StdFlight1 & $\begin{array}{c}\text { Standard deviation of } \\
\text { Flight1 in a word. }\end{array}$ \\
\hline StdFlight2 & $\begin{array}{c}\text { Standard deviation of } \\
\text { Flight2 in a word. }\end{array}$ \\
\hline StdFlight3 & $\begin{array}{c}\text { Standard deviation of } \\
\text { Flight3 in a word. }\end{array}$ \\
\hline StdFlight4 & $\begin{array}{c}\text { Standard deviation of } \\
\text { Flight4 in a word. }\end{array}$ \\
\hline
\end{tabular}

Table 4. Proposed Features Extracted from the Same Example String "this is that"

\begin{tabular}{|c|c|c|}
\hline Feature & word: "this" & word: "that" \\
\hline WordHold & $\left(s_{1 R}-t_{1 P}\right)$ & $\left(t_{3 R}-t_{2 P}\right)$ \\
\hline AvgKeyHold & $\begin{array}{c}\operatorname{Avg}\left[\left(t_{1 R}-t_{1 P}\right),\left(h_{1 R}-h_{1 P}\right)\right. \\
\left.\left(i_{1 R}-i_{1 P}\right),\left(s_{1 R}-s_{1 P}\right)\right]\end{array}$ & $\begin{array}{c}\operatorname{Avg}\left[\left(t_{2 R}-t_{2 P}\right),\left(h_{2 R}-h_{2 P}\right)\right. \\
\left.\quad\left(a_{1 R}-a_{1 P}\right),\left(t_{3 R}-t_{3 P}\right)\right]\end{array}$ \\
\hline AvgFlight1 & $\begin{array}{c}\operatorname{Avg}\left[\left(h_{1 P}-t_{1 R}\right),\left(i_{1 P}-h_{1 R}\right),\right. \\
\left.\left(s_{1 P}-i_{1 R}\right)\right]\end{array}$ & $\begin{array}{c}\operatorname{Avg}\left[\left(h_{2 P}-t_{2 R}\right),\left(a_{2 P}-h_{2 R}\right),\right. \\
\left.\left(t_{1 P}-a_{1 R}\right)\right]\end{array}$ \\
\hline AvgFlight2 & $\begin{array}{c}\operatorname{Avg}\left[\left(h_{1 R}-t_{1 R}\right),\left(i_{1 R}-h_{1 R}\right),\right. \\
\left.\left(s_{1 R}-i_{1 R}\right)\right]\end{array}$ & $\begin{array}{c}\operatorname{Avg}\left[\left(h_{2 R}-t_{2 R}\right),\left(a_{2 R}-h_{2 R}\right),\right. \\
\left.\left(t_{1 R}-a_{1 R}\right)\right]\end{array}$ \\
\hline AvgFlight3 & $\begin{array}{c}\operatorname{Avg}\left[\left(h_{1 P}-t_{1 P}\right),\left(i_{1 P}-h_{1 P}\right),\right. \\
\left.\left(s_{1 P}-i_{1 P}\right)\right]\end{array}$ & $\begin{array}{c}\operatorname{Avg}\left[\left(h_{2 P}-t_{2 P}\right),\left(a_{2 P}-h_{2 P}\right),\right. \\
\left.\left(t_{1 P}-a_{1 P}\right)\right]\end{array}$ \\
\hline AvgFlight4 & $\begin{array}{c}\operatorname{Avg}\left[\left(h_{1 R^{-}} t_{1 P}\right),\left(i_{1 R}-h_{1 P}\right),\right. \\
\left(s_{\left.\left.1 R^{-}-i_{1 P}\right)\right]}\right.\end{array}$ & $\begin{array}{c}\operatorname{Avg}\left[\left(h_{2 R}-t_{2 P}\right),\left(a_{2 R}-h_{2 P}\right),\right. \\
\left.\left(t_{1 R}-a_{1 P}\right)\right]\end{array}$ \\
\hline StdKeyHold & $\begin{array}{l}\operatorname{Std}\left[\left(t_{1 R}-t_{1 P}\right),\left(h_{1 R}-h_{1 P}\right)\right. \\
\left.\quad\left(i_{1 R}-i_{1 P}\right),\left(s_{1 R}-s_{1 P}\right)\right]\end{array}$ & $\begin{array}{l}\operatorname{Std}\left[\left(t_{2 R}-t_{2 P}\right),\left(h_{2 R}-h_{2 P}\right),\right. \\
\left.\quad\left(a_{1 R}-a_{1 P}\right),\left(t_{3 R}-t_{3 P}\right)\right]\end{array}$ \\
\hline StdFlight1 & $\begin{array}{c}\operatorname{Std}\left[\left(h_{1 P}-t_{1 R}\right),\left(i_{1 P}-h_{1 R}\right)\right. \\
\left.\left(s_{1 P}-i_{1 R}\right)\right]\end{array}$ & $\begin{array}{c}\operatorname{Std}\left[\left(h_{2 P}-t_{2 R}\right),\left(a_{2 P}-h_{2 R}\right),\right. \\
\left.\left(t_{1 P}-a_{1 R}\right)\right]\end{array}$ \\
\hline StdFlight2 & $\begin{array}{c}\operatorname{Std}\left[\left(h_{1 R}-t_{1 R}\right),\left(i_{1 R}-h_{1 R}\right)\right. \\
\left.\left(s_{1 R}-i_{1 R}\right)\right]\end{array}$ & $\begin{array}{c}\operatorname{Std}\left[\left(h_{2 R}-t_{2 R}\right),\left(a_{2 R}-h_{2 R}\right),\right. \\
\left.\left(t_{1 R}-a_{1 R}\right)\right]\end{array}$ \\
\hline StdFlight3 & $\begin{array}{c}\operatorname{Std}\left[\left(h_{1 P}-t_{1 P}\right),\left(i_{1 P}-h_{1 P}\right)\right. \\
\left.\left(s_{1 P}-i_{1 P}\right)\right]\end{array}$ & $\begin{array}{c}\operatorname{Std}\left[\left(h_{2 P}-t_{2 P}\right),\left(a_{2 P}-h_{2 P}\right)\right. \\
\left.\left(t_{1 P}-a_{1 P}\right)\right]\end{array}$ \\
\hline StdFlight4 & $\begin{array}{c}\operatorname{Std}\left[\left(h_{1 R}-t_{1 P}\right),\left(i_{1 R}-h_{1 P}\right)\right. \\
\left(s_{\left.\left.1 R^{-}-i_{1 P}\right)\right]}\right.\end{array}$ & $\begin{array}{c}\operatorname{Std}\left[\left(h_{2 R}-t_{2 P}\right),\left(a_{2 R}-h_{2 P}\right),\right. \\
\left(t_{\left.\left.1 R^{-}-a_{1 P}\right)\right]}\right.\end{array}$ \\
\hline
\end{tabular}

$t_{1 R}$ stands for release of key $t_{1}$ (the subscript 1 stands for the first occurrence of "t") and $t_{1 P}$ stands for press of key $t_{1}$ and so on, Avg and Std stand for average and standard deviation, respectively.

discriminability of both conventional and proposed features and try and gain insights on why these features offer different levels of precision for user identification.

\section{DATA COLLECTION}

Figure 2 summarizes the data collection process. After IRB approval from our university, the data collection exercise was carried out. We use the data from 20 users in this study. Emails were sent out to all students, faculty, and staff to procure the participant population. All participants were proficient in English. Unlike most other studies, we did not restrict the type of device or text in our experiments. Each participant performed a set of common day-to-day activities on three different devices: a desktop, a tablet, and a phone. Desktop I/O consisted of a standard QWERTY keyboard, optic mouse, and 21" monitor. HTC-Nexus-9 tablets, Samsung-S6, and HTC-One phones were used in the process of data collection. The Samsung Galaxy S6 had a screen size of 5.1 inches with body dimensions of $143.4 \times 70.5 \times 6.8 \mathrm{~mm}$ and weighing $138 \mathrm{~g}$, whereas the HTC-One had a screen size of 5.0 inches with body dimensions of $146.4 \times 70.6 \times 9.4 \mathrm{~mm}$ and weighing $160 \mathrm{~g}$. As the default Android keyboard does not allow logging of keystrokes, we created and used an Android QWERTY keyboard on screen that was similar to the default Android QWERTY keyboard. The phones and tablets were locked in portrait orientation and users were allowed to type on them with any comfortable posture that they preferred; there were no restrictions on the holding style of the phone and all participants chose to hold the phone with both hands while typing. For the typing activities, both free text (spontaneous or unscripted typing) and fixed text (predetermined words or sentences to be typed as is) were used as a real-life situation would include a mix of them. 


\begin{tabular}{cccc}
\multirow{2}{*}{ Tasks } & \multicolumn{3}{c}{ Transcription (Fixed Text) } \\
\cline { 2 - 4 } & $\begin{array}{c}\text { Browsing (Free Text) } \\
\text { Q \& A (Free Text) }\end{array}$ & $\mathrm{Q} \& \mathrm{~A}$ (Free Text) & $\mathrm{Q} \&$ A (Free Text) \\
\hline Approx. Duration & 45 mins. & 25 mins. & 25 mins. \\
\hline $\begin{array}{c}\text { Approx. Keystrokes } \\
\text { per participant }\end{array}$ & 12,500 & 9,000 & 10,000 \\
\hline
\end{tabular}

Fig. 2. Highlights of our Data Collection effort.

For the desktop section of data collection, participants were asked to first type fixed text that consisted of two sentences, 20 times (Appendix E). This was followed by a brief session on browsing the Internet with tasks that approximated shopping behavior such as searching for the best prices and simultaneously making notes. The participants were then asked to type their free-text answers to 10 questions with varying cognitive loads [10], as shown in Appendices B, C, and D. Keystroke events such as press, release, and their corresponding timestamps were recorded using windows keyboard hooks during the entire activity. For the tablet and phone section of the data collection, participants were asked to first type the same fixed text, 20 times. This was followed by typing free-text answers to a set of 10 questions with different cognitive loads. The questions used in each section were different. Keystroke and touch events with their corresponding timestamps were logged during the entire duration for both hand-held devices. The participants took about 45 minutes to complete the tasks on the desktop and 25 minutes each on the tablet and phone. Each participant had approximately $12.5 \mathrm{~K}$ keystrokes on the desktop, 9K keystrokes on the tablet, and $10 \mathrm{~K}$ keystrokes on the phone.

\section{FEATURE DISCRIMINABILITY ANALYSIS}

To analyze the discriminative power of features, we first model an estimate of probability density function (PDF), given by the histograms of the values for each feature. We use fixed bin size of $1 \mathrm{~ms}$ for all histograms to match the clock resolution used to record keystroke events. To determine the discriminative potential of features, we find the overlap between the PDFs using the Bhattacharyya distance metric as described by Sim and Janakiraman [48]:

$$
\operatorname{Dist}_{B}(H 1, H 2)=\sum_{x=1}^{n b i n s} \sqrt{H 1(x) H 2(x)} .
$$

Equation (1) defines the Bhattacharyya distance between two PDFs, $H 1(x)$ and $H 2(x)$, in which the distance lies between 0 and 1 . A Dist ${ }_{B}$ value of 0 implies no overlap in the PDFs, hence maximum discriminability and 1 implies complete overlap in the PDFs, hence minimum discriminability. As the PDFs we deal with are discretized, to implement Equation (1), we simply multiply the probability of the corresponding bins, take the product's positive square root, and sum it over all bins of the PDFs. Figure 3 shows an example of the computation of Dist ${ }_{B}$ values, using the PDFs of two random users for KeyHold values of the character " $t$ " on different devices. Figure 3(a) shows the PDFs KeyHold of " $\mathrm{t}$ " for a desktop; the amount of overlap between the two is reflected by the Dist $_{B}$ computed, which is 0.487 . Similarly, Figures 3(b) and 3(c) show the PDFs on a tablet and phone that have Dist $_{B}$ of 0.733 and 0.754 , respectively. As explained in Reference [48], a large Dist $t_{B}$ 


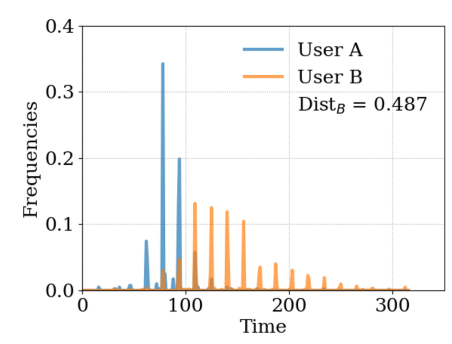

(a) KeyHold of "t" on desktop

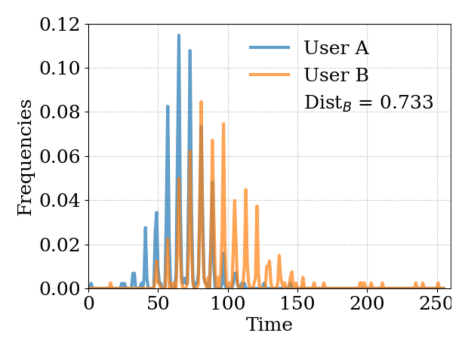

(b) KeyHold of "t" on tablet

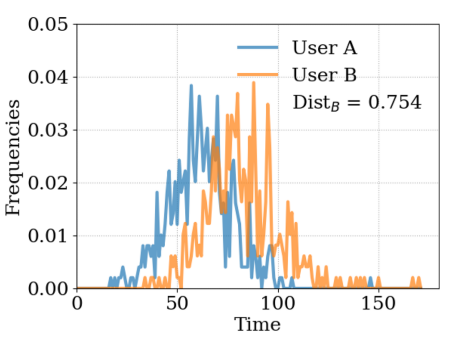

(c) KeyHold of " $\mathrm{t}$ " on phone

Fig. 3. Example of Dist $_{B}$ computation: Histograms representing the probability density functions of KeyHold values for the character "t," for Users A and B on (a) desktop, (b) tablet, and (c) phone along with their corresponding Bhattacharyya distance.

Table 5. The Inter-User Dist $t_{B}$ Values for KeyHold Distributions on all Devices

\begin{tabular}{|c|l|l|l|l|l|l|l|l|l|}
\hline Device & \multicolumn{3}{|c|}{ Desktop } & \multicolumn{3}{c|}{ Tablet } & \multicolumn{3}{c|}{ Phone } \\
\hline \hline Uni-Graph & Mean & StD & Median & Mean & StD & Median & Mean & StD & Median \\
\hline space & 0.785 & 0.103 & 0.8 & 0.792 & 0.145 & 0.837 & 0.7 & 0.191 & 0.731 \\
\hline bspace & 0.723 & 0.069 & 0.728 & 0.667 & 0.117 & 0.687 & 0.541 & 0.174 & 0.553 \\
\hline $\mathrm{a}$ & 0.71 & 0.123 & 0.745 & 0.688 & 0.175 & 0.737 & 0.6 & 0.207 & 0.64 \\
\hline $\mathrm{e}$ & 0.777 & 0.128 & 0.812 & 0.776 & 0.162 & 0.835 & 0.723 & 0.156 & 0.739 \\
\hline $\mathrm{h}$ & 0.738 & 0.141 & 0.777 & 0.741 & 0.102 & 0.761 & 0.559 & 0.176 & 0.562 \\
\hline $\mathrm{i}$ & 0.73 & 0.121 & 0.753 & 0.81 & 0.084 & 0.834 & 0.626 & 0.18 & 0.65 \\
\hline $\mathrm{l}$ & 0.784 & 0.091 & 0.796 & 0.786 & 0.094 & 0.803 & 0.568 & 0.188 & 0.586 \\
\hline $\mathrm{n}$ & 0.693 & 0.149 & 0.729 & 0.802 & 0.095 & 0.832 & 0.588 & 0.194 & 0.6 \\
\hline $\mathrm{o}$ & 0.711 & 0.131 & 0.737 & 0.809 & 0.084 & 0.833 & 0.602 & 0.189 & 0.615 \\
\hline $\mathrm{r}$ & 0.665 & 0.139 & 0.701 & 0.732 & 0.132 & 0.767 & 0.61 & 0.171 & 0.591 \\
\hline $\mathrm{s}$ & 0.761 & 0.116 & 0.789 & 0.775 & 0.138 & 0.832 & 0.659 & 0.18 & 0.673 \\
\hline $\mathrm{t}$ & 0.75 & 0.135 & 0.784 & 0.792 & 0.112 & 0.824 & 0.682 & 0.157 & 0.685 \\
\hline
\end{tabular}

implies that the Bayes' error is large, and a small Dist $_{B}$ value could lead to small Bayes' error. We use $\operatorname{Dist}_{B}$ as a measure to analyze how well a feature separates the users from each other.

\section{ANALYSIS OF CONVENTIONAL KD FEATURES}

To analyze the discriminability of conventional KD features, we use the 12 most occurring Unigraphs and 25 most occurring Di-graphs in our dataset. These Uni-graphs and Di-graphs are shown in Tables 5 and 6 and include "space" and "backspace." For Uni-graphs and Di-graphs of each user, we extract feature-specific PDFs (one for each conventional KD feature in discussion). Once the PDFs are computed on all three devices, we calculate Dist $_{B}$ between corresponding PDFs for each pair of users and use the mean, standard deviation, and median to gain insight into the properties of these features.

\subsection{KeyHold}

Description: KeyHold is the time duration for which a key is held down for one instance of the key (one press to one release of the same key).

Inference: As values in Table 5 show, the mean values of Dist $_{B}$ for KeyHold are too high for all devices, implying that this is not a very discriminative feature. Most mean Dist $_{B}$ values for 
Table 6. The Inter-User Dist $_{B}$ Values Flight1 Distributions on All Devices

\begin{tabular}{|c|c|c|c|c|c|c|c|c|c|}
\hline Device & \multicolumn{3}{|c|}{ Desktop } & \multicolumn{3}{|c|}{ Tablet } & \multicolumn{3}{|c|}{ Phone } \\
\hline Di-Graph & Mean & StD & Median & Mean & StD & Median & Mean & StD & Median \\
\hline (“space," “a”) & 0.495 & 0.134 & 0.505 & 0.104 & 0.06 & 0.098 & 0.086 & 0.064 & 0.071 \\
\hline ("space," "i") & 0.427 & 0.159 & 0.425 & 0.093 & 0.056 & 0.084 & 0.071 & 0.061 & 0.054 \\
\hline ("space," "s") & 0.526 & 0.12 & 0.54 & 0.135 & 0.064 & 0.128 & 0.081 & 0.056 & 0.065 \\
\hline ("space," "t") & 0.594 & 0.135 & 0.614 & 0.193 & 0.066 & 0.186 & 0.146 & 0.081 & 0.133 \\
\hline ("bspace," "bspace") & 0.576 & 0.1 & 0.581 & 0.51 & 0.1 & 0.512 & 0.417 & 0.174 & 0.427 \\
\hline (“e," “space”) & 0.554 & 0.194 & 0.55 & 0.436 & 0.165 & 0.47 & 0.308 & 0.16 & 0.303 \\
\hline (“e," “n”) & \multicolumn{3}{|c|}{-} & 0.256 & 0.137 & 0.257 & 0.181 & 0.121 & 0.169 \\
\hline (“e," “r”) & 0.384 & 0.23 & 0.37 & 0.393 & 0.152 & 0.409 & 0.287 & 0.143 & 0.278 \\
\hline ("e," "s") & 0.555 & 0.171 & 0.587 & 0.429 & 0.154 & 0.445 & 0.331 & 0.137 & 0.333 \\
\hline ("n," "space") & 0.405 & 0.211 & 0.429 & 0.361 & 0.136 & 0.357 & 0.217 & 0.139 & 0.192 \\
\hline (“o," "space”) & 0.379 & 0.241 & 0.345 & 0.275 & 0.13 & 0.264 & 0.188 & 0.133 & 0.168 \\
\hline (“o," “n”) & 0.412 & 0.219 & 0.464 & 0.353 & 0.158 & 0.385 & 0.269 & 0.14 & 0.253 \\
\hline ("r," "e") & 0.434 & 0.27 & 0.465 & 0.472 & 0.145 & 0.492 & 0.378 & 0.154 & 0.371 \\
\hline (“s," "space") & 0.499 & 0.206 & 0.501 & 0.363 & 0.142 & 0.378 & 0.228 & 0.141 & 0.227 \\
\hline ("s," "e") & 0.531 & 0.234 & 0.572 & 0.59 & 0.124 & 0.62 & 0.39 & 0.159 & 0.38 \\
\hline ("t," "space") & 0.481 & 0.223 & 0.465 & 0.325 & 0.113 & 0.34 & 0.248 & 0.127 & 0.232 \\
\hline (“t," e") & \multicolumn{3}{|c|}{-} & 0.267 & 0.132 & 0.253 & 0.237 & 0.135 & 0.223 \\
\hline ("t," "h") & 0.511 & 0.242 & 0.523 & 0.462 & 0.158 & 0.5 & 0.344 & 0.152 & 0.357 \\
\hline (“a," "r") & 0.399 & 0.231 & 0.407 & 0.261 & 0.124 & 0.247 & \multicolumn{3}{|c|}{-} \\
\hline (“t," "o") & 0.43 & 0.205 & 0.392 & \multicolumn{3}{|c|}{-} & & - & \\
\hline (“space,” "w”) & 0.351 & 0.159 & 0.365 & \multicolumn{3}{|c|}{-} & \multicolumn{3}{|c|}{ - } \\
\hline ("h," "e") & 0.514 & 0.229 & 0.537 & \multicolumn{3}{|c|}{ - } & \multicolumn{3}{|c|}{ - } \\
\hline (“i," "n") & 0.346 & 0.218 & 0.361 & \multicolumn{3}{|c|}{-} & \multicolumn{3}{|c|}{-} \\
\hline (“1," “e”) & 0.478 & 0.22 & 0.512 & \multicolumn{3}{|c|}{ 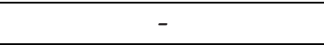 } & \multicolumn{3}{|c|}{ - } \\
\hline (“l," “l”) & 0.671 & 0.216 & 0.762 & \multicolumn{3}{|c|}{ - } & \multicolumn{3}{|c|}{-} \\
\hline
\end{tabular}

desktop lie around 0.7 to 0.78 , which are hinting at very high overlap among the PDFs. The least and highest mean Dist $_{B}$ were for "n" and "space" with 0.693 and 0.785 , respectively. For tablet, the values $\operatorname{Dist}_{B}$ are very high, in the range of 0.7 to 0.8 for most characters. The least and highest mean Dist $_{B}$ were "backspace" $=.667$ and "o" $=.809$, respectively. In the case of phone, we see a very negligible reduction in mean Dist $_{B}$ values for phone, with most values between 0.58 to 0.72 , which are still very high. The least and highest mean Dist $_{B}$ were "backspace" $=0.54$ and "e" $=0.72$, respectively. By these values, we can infer that KeyHold is not a discriminable feature on any of these devices.

\subsection{Flight 1}

Description: For a Di-graph, Flight1 is the time duration between release of the first key and press for the second key.

Inference: Table 6 shows the mean, standard deviation, and median of the Dist $_{B}$ values for Flight1 on all three devices for the selected 25 most-common Di-graphs. Clearly, the mean values of Dist $_{B}$ for Flight1 are considerably better than those for KeyHold on all devices. We observe that most mean Dist $_{B}$ values for desktop lie around 0.3 to 0.6 , which is still not desirable for a feature meant to be discriminative. The least and highest mean Dist $_{B}$ were for the Di-graphs 
Table 7. The Inter-User Dist $_{B}$ Values for Flight2 Distributions on All Devices

\begin{tabular}{|c|c|c|c|c|c|c|c|c|c|}
\hline Device & \multicolumn{3}{|c|}{ Desktop } & \multicolumn{3}{|c|}{ Tablet } & \multicolumn{3}{|c|}{ Phone } \\
\hline Di-Graph & Mean & StD & Median & Mean & $\overline{\mathrm{StD}}$ & Median & Mean & $\overline{\mathrm{StD}}$ & Median \\
\hline (“space,” “a”) & 0.511 & 0.096 & 0.518 & 0.097 & 0.055 & 0.086 & 0.083 & 0.061 & 0.074 \\
\hline (“space," "i”) & 0.484 & 0.125 & 0.497 & 0.087 & 0.057 & 0.074 & 0.067 & 0.05 & 0.055 \\
\hline ("space," "s") & 0.556 & 0.109 & 0.563 & 0.119 & 0.057 & 0.107 & 0.078 & 0.051 & 0.071 \\
\hline ("space," "t") & 0.668 & 0.109 & 0.703 & 0.181 & 0.074 & 0.184 & 0.141 & 0.08 & 0.133 \\
\hline ("bspace," "bspace”) & 0.699 & 0.094 & 0.713 & 0.582 & 0.164 & 0.602 & 0.517 & 0.186 & 0.55 \\
\hline (“e," "space") & 0.679 & 0.126 & 0.695 & 0.362 & 0.131 & 0.38 & 0.276 & 0.151 & 0.27 \\
\hline (“e,”“n”) & \multicolumn{3}{|c|}{-} & 0.23 & 0.128 & 0.246 & 0.136 & 0.113 & 0.108 \\
\hline (“e,” “r”) & 0.502 & 0.157 & 0.528 & 0.341 & 0.179 & 0.336 & 0.275 & 0.159 & 0.274 \\
\hline (“e," "s") & 0.586 & 0.144 & 0.624 & 0.37 & 0.159 & 0.383 & 0.275 & 0.139 & 0.271 \\
\hline (“n," "space") & 0.466 & 0.192 & 0.49 & 0.308 & 0.125 & 0.317 & 0.177 & 0.135 & 0.162 \\
\hline (“o," "space") & 0.475 & 0.229 & 0.502 & 0.235 & 0.109 & 0.247 & 0.176 & 0.133 & 0.154 \\
\hline (“o,” “n”) & 0.453 & 0.156 & 0.465 & 0.305 & 0.146 & 0.332 & 0.22 & 0.144 & 0.214 \\
\hline ("r," "e") & 0.563 & 0.195 & 0.621 & 0.455 & 0.145 & 0.472 & 0.356 & 0.175 & 0.342 \\
\hline (“s," “space”) & 0.59 & 0.168 & 0.611 & 0.293 & 0.11 & 0.298 & 0.211 & 0.141 & 0.202 \\
\hline (“s," "e") & 0.599 & 0.185 & 0.64 & 0.538 & 0.153 & 0.572 & 0.371 & 0.176 & 0.357 \\
\hline ("t," "space”) & 0.556 & 0.161 & 0.576 & 0.264 & 0.095 & 0.271 & 0.223 & 0.111 & 0.204 \\
\hline ("t," "e") & \multicolumn{3}{|c|}{-} & 0.264 & 0.109 & 0.254 & 0.208 & 0.126 & 0.187 \\
\hline ("t," "h") & 0.615 & 0.18 & 0.657 & 0.439 & 0.144 & 0.453 & 0.294 & 0.154 & 0.286 \\
\hline (“a," “r”) & 0.49 & 0.179 & 0.522 & 0.224 & 0.114 & 0.22 & \multicolumn{3}{|c|}{-} \\
\hline (“t," "o") & 0.548 & 0.145 & 0.56 & \multicolumn{3}{|c|}{-} & & - & \\
\hline (“space," "w”) & 0.382 & 0.143 & 0.395 & \multicolumn{3}{|c|}{-} & \multicolumn{3}{|c|}{-} \\
\hline (“h,”"e") & 0.646 & 0.114 & 0.661 & \multicolumn{3}{|c|}{-} & \multicolumn{3}{|c|}{-} \\
\hline (“i," "n") & 0.411 & 0.187 & 0.436 & \multicolumn{3}{|c|}{-} & \multicolumn{3}{|c|}{-} \\
\hline ("l," "e") & 0.6 & 0.109 & 0.615 & \multicolumn{3}{|c|}{-} & \multicolumn{3}{|c|}{-} \\
\hline$(" 1, "$ "1”) & 0.639 & 0.169 & 0.692 & \multicolumn{3}{|c|}{ - } & \multicolumn{3}{|c|}{-} \\
\hline
\end{tabular}

$(\mathrm{i}, \mathrm{n})=0.346$ and $(1, \mathrm{l})=0.671$, respectively. The mean Dist $_{B}$ values for tablet are considerably low for a few Di-graphs, which is very desirable, but as these are a negligible minority (space: a,i,s,t), their scope of extraction is largely reduced. The least and highest mean Dist $_{B}$ were for the Digraphs (space,i) $=0.093$ and $(\mathrm{s}, \mathrm{e})=0.59$, respectively. We observe the mean Dist $_{B}$ values on phone to be similar to tablet with a small minority (space: a,i,s,t) $(\mathrm{e}, \mathrm{n})$ of Di-graphs having low Dist $t_{B}$ values. (space,i) has the least mean value at 0.071 while (Backspace, Backspace) has the highest at 0.417. Overall, Flight1 does not seem to be a very discriminative feature. As all Di-graphs have very high values of mean Dist $_{B}$ on desktop and very few values in tablet and phone are at a desirable range, this feature is not a good feature to provide separation in user keystroke data.

\subsection{Flight2}

Description: For a Di-graph, Flight2 is the time duration between release of the first key and release of the second key.

Inferences: Table 7 shows the mean Dist $_{B}$ values for Flight 2 on all three devices for the selected 25 common Di-graphs. The mean values of Dist $t_{B}$ for Flight2 are considerably better than those for KeyHold on all devices, but are similar to Flight1. Most mean Dist $_{B}$ values for desktop lie around 0.3 to 0.6 , the least and highest mean Dist $_{B}$ values being (space,w) $=0.382$ and 
Table 8. The Inter-User Dist ${ }_{B}$ Values for Flight3 Distributions on all Devices

\begin{tabular}{|c|c|c|c|c|c|c|c|c|c|}
\hline Device & \multicolumn{3}{|c|}{ Desktop } & \multicolumn{3}{|c|}{ Tablet } & \multicolumn{3}{|c|}{ Phone } \\
\hline Di-Graph & Mean & StD & Median & Mean & StD & Median & Mean & StD & $\overline{\text { Median }}$ \\
\hline (“space,” “a”) & 0.542 & 0.092 & 0.549 & 0.131 & 0.08 & 0.12 & 0.08 & 0.057 & 0.068 \\
\hline (“space," "i") & 0.495 & 0.144 & 0.515 & 0.109 & 0.072 & 0.089 & 0.066 & 0.049 & 0.056 \\
\hline ("space," "s") & 0.58 & 0.105 & 0.594 & 0.15 & 0.078 & 0.146 & 0.078 & 0.051 & 0.069 \\
\hline ("space," "t") & 0.655 & 0.113 & 0.685 & 0.224 & 0.099 & 0.221 & 0.141 & 0.077 & 0.127 \\
\hline ("bspace," "bspace") & 0.593 & 0.119 & 0.586 & 0.584 & 0.154 & 0.613 & 0.517 & 0.187 & 0.558 \\
\hline ("e," "space") & 0.708 & 0.081 & 0.714 & 0.431 & 0.152 & 0.459 & 0.296 & 0.144 & 0.315 \\
\hline (“e,” “n”) & \multicolumn{3}{|c|}{-} & 0.251 & 0.138 & 0.249 & 0.146 & 0.107 & 0.146 \\
\hline (“e," "r") & 0.531 & 0.165 & 0.559 & 0.32 & 0.166 & 0.304 & 0.242 & 0.142 & 0.249 \\
\hline (“e,” “s”) & 0.643 & 0.089 & 0.654 & 0.387 & 0.175 & 0.396 & 0.275 & 0.12 & 0.272 \\
\hline ("n," "space") & 0.508 & 0.167 & 0.547 & 0.35 & 0.132 & 0.342 & 0.154 & 0.113 & 0.126 \\
\hline ("o," "space") & 0.524 & 0.211 & 0.578 & 0.305 & 0.146 & 0.291 & 0.173 & 0.121 & 0.161 \\
\hline (“o,” “n”) & 0.493 & 0.179 & 0.532 & 0.3 & 0.147 & 0.306 & 0.216 & 0.112 & 0.221 \\
\hline ("r," "e") & 0.652 & 0.17 & 0.699 & 0.457 & 0.141 & 0.463 & 0.352 & 0.149 & 0.364 \\
\hline (“s," "space") & 0.639 & 0.113 & 0.656 & 0.377 & 0.133 & 0.39 & 0.209 & 0.134 & 0.197 \\
\hline ("s," "e") & 0.639 & 0.151 & 0.659 & 0.537 & 0.176 & 0.563 & 0.382 & 0.16 & 0.382 \\
\hline ("t," "space") & 0.604 & 0.14 & 0.631 & 0.321 & 0.121 & 0.314 & 0.232 & 0.113 & 0.219 \\
\hline ("t," "e") & \multicolumn{3}{|c|}{-} & 0.259 & 0.124 & 0.243 & 0.178 & 0.12 & 0.158 \\
\hline (“t," "h") & 0.654 & 0.151 & 0.691 & 0.49 & 0.122 & 0.495 & 0.297 & 0.141 & 0.307 \\
\hline ("a," "r") & 0.525 & 0.153 & 0.558 & 0.241 & 0.125 & 0.221 & \multicolumn{3}{|c|}{-} \\
\hline (“t," "o") & 0.522 & 0.159 & 0.516 & \multicolumn{3}{|c|}{-} & \multirow{2}{*}{\multicolumn{3}{|c|}{-}} \\
\hline ("space," "w”) & 0.397 & 0.151 & 0.414 & \multicolumn{3}{|c|}{-} & & & \\
\hline ("h,”"e") & 0.618 & 0.13 & 0.635 & \multicolumn{3}{|c|}{$\begin{array}{lll}- & - \\
-\end{array}$} & \multicolumn{3}{|c|}{ - } \\
\hline (“i," "n”) & 0.423 & 0.191 & 0.469 & \multicolumn{3}{|c|}{-} & \multicolumn{3}{|c|}{-} \\
\hline (“1," “e”) & 0.579 & 0.131 & 0.592 & \multicolumn{3}{|c|}{-} & \multicolumn{3}{|c|}{-} \\
\hline ("1," "1") & 0.633 & 0.194 & 0.7 & \multicolumn{3}{|c|}{ - } & \multicolumn{3}{|c|}{-} \\
\hline
\end{tabular}

$($ space, $t)=0.668$, respectively. For the tablet, we observe that the mean Dist $_{B}$ values for a few Digraphs are considerably low, which is very desirable, but again, as these are a negligible minority (space: a,i,s,t), their scope of extraction is largely reduced: the least mean $\operatorname{Dist}_{B}$ being $($ space,i) = 0.097 and highest being (backspace,backspace) $=0.582$. We observe phone to be similar to tablet with a small number (space: a,i,s,t) (e,n) of Di-graphs having low mean Dist $_{B}$ values: the least and highest Dist $_{B}$ values being $($ space, $\mathrm{i})=0.067$ and $($ Backspace,Backspace $)=0.517$, respectively. Overall, we infer that Flight2 is not a discriminative feature. As all Di-graphs have very high mean Dist $_{B}$ values on the desktop and very few values in tablet and phone are desirable, this feature is not a good feature for separation of users based on keystroke data.

\subsection{Flight3}

Description: For a Di-graph, Flight3 is the time duration between press of the first key and press of the second key.

Inference: As shown in Table 8, mean Dist $_{B}$ values for Flight3 on all three devices show that Flight3 is considerably more discriminative than KeyHold on all devices, but is similar to Flight1 and Flight2. Most mean Dist $_{B}$ values for desktop lie around 0.49 to 0.63 , which is still high. The least and highest mean $\operatorname{Dist}_{B}$ values were for the Di-Graphs (space,w) $=0.397$ and 
Table 9. The Inter-User Dist $_{B}$ Values for Flight4 Distributions on All Devices

\begin{tabular}{|c|c|c|c|c|c|c|c|c|c|}
\hline Device & \multicolumn{3}{|c|}{ Desktop } & \multicolumn{3}{|c|}{ Tablet } & \multicolumn{3}{|c|}{ Phone } \\
\hline Di-Graph & Mean & StD & Median & Mean & StD & Median & Mean & StD & $\overline{~ M e d i a n}$ \\
\hline (“space,” “a”) & 0.514 & 0.089 & 0.522 & 0.106 & 0.064 & 0.099 & 0.083 & 0.056 & 0.077 \\
\hline (“space," "i") & 0.498 & 0.12 & 0.506 & 0.098 & 0.059 & 0.088 & 0.06 & 0.043 & 0.053 \\
\hline ("space," "s") & 0.573 & 0.092 & 0.58 & 0.131 & 0.068 & 0.122 & 0.073 & 0.051 & 0.063 \\
\hline ("space," "t") & 0.671 & 0.096 & 0.69 & 0.199 & 0.09 & 0.185 & 0.137 & 0.076 & 0.132 \\
\hline ("bspace," "bspace") & 0.579 & 0.1 & 0.593 & 0.421 & 0.138 & 0.432 & 0.34 & 0.178 & 0.345 \\
\hline (“e," "space”) & 0.74 & 0.071 & 0.75 & 0.336 & 0.118 & 0.355 & 0.267 & 0.141 & 0.268 \\
\hline (“e,”"n”) & \multicolumn{3}{|c|}{-} & 0.218 & 0.12 & 0.214 & 0.122 & 0.098 & 0.105 \\
\hline (“e," "r") & 0.576 & 0.122 & 0.589 & 0.285 & 0.168 & 0.288 & 0.215 & 0.139 & 0.204 \\
\hline (“e," “s”) & 0.639 & 0.091 & 0.646 & 0.322 & 0.149 & 0.333 & 0.223 & 0.126 & 0.216 \\
\hline ("n," "space") & 0.533 & 0.164 & 0.561 & 0.273 & 0.116 & 0.278 & 0.147 & 0.122 & 0.121 \\
\hline ("o," "space") & 0.56 & 0.17 & 0.594 & 0.217 & 0.107 & 0.213 & 0.155 & 0.115 & 0.145 \\
\hline (“o,” “n”) & 0.511 & 0.121 & 0.513 & 0.262 & 0.125 & 0.267 & 0.177 & 0.121 & 0.182 \\
\hline ("r," "e") & 0.717 & 0.105 & 0.734 & 0.424 & 0.144 & 0.438 & 0.303 & 0.154 & 0.324 \\
\hline (“s," “space”) & 0.652 & 0.105 & 0.662 & 0.272 & 0.098 & 0.276 & 0.184 & 0.123 & 0.173 \\
\hline (“s," "e") & 0.703 & 0.103 & 0.72 & 0.46 & 0.165 & 0.489 & 0.342 & 0.178 & 0.344 \\
\hline ("t," "space") & 0.642 & 0.102 & 0.647 & 0.255 & 0.098 & 0.252 & 0.221 & 0.102 & 0.218 \\
\hline ("t," "e") & \multicolumn{3}{|c|}{-} & 0.239 & 0.102 & 0.235 & 0.167 & 0.113 & 0.148 \\
\hline (“t," "h") & 0.671 & 0.137 & 0.704 & 0.451 & 0.116 & 0.458 & 0.257 & 0.15 & 0.258 \\
\hline (“a,” “r”) & 0.509 & 0.161 & 0.549 & 0.21 & 0.116 & 0.203 & \multicolumn{3}{|c|}{-} \\
\hline (“t," “o") & 0.572 & 0.142 & 0.585 & \multicolumn{3}{|c|}{-} & & - & \\
\hline (“space,” “w”) & 0.4 & 0.145 & 0.407 & \multicolumn{3}{|c|}{ - } & \multicolumn{3}{|c|}{-} \\
\hline (“h,”"e") & 0.65 & 0.108 & 0.679 & \multicolumn{3}{|c|}{-} & \multicolumn{3}{|c|}{-} \\
\hline (“i," "n”) & 0.477 & 0.151 & 0.51 & \multicolumn{3}{|c|}{-} & \multicolumn{3}{|c|}{-} \\
\hline (“l," “e”) & 0.627 & 0.107 & 0.647 & \multicolumn{3}{|c|}{ - } & \multicolumn{3}{|c|}{-} \\
\hline (“1," “l”) & 0.625 & 0.166 & 0.67 & \multicolumn{3}{|c|}{ - } & \multicolumn{3}{|c|}{-} \\
\hline
\end{tabular}

(e,space $)=0.708$, respectively. For tablet, the mean Dist $_{B}$ values for a few Di-graphs are considerably low, similar to Flight1 and Flight2, which is very desirable, but as these are a negligible minority (space: a,i,s,t), their scope of extraction is largely reduced. The least and highest values for Dist $_{B}$ were $($ space,, $\mathrm{i})=0.109$ and $($ backspace,backspace $)=0.584$, respectively. We observe phone to be similar to tablet with a small minority (space: a,i,s,t) $(\mathrm{e}, \mathrm{n})$ of Di-graphs having low Dist $_{B}$ values: the lowest Dist $_{B}$ value being (space,i) $=0.066$ and highest being (Backspace, Backspace) $=0.517$. We infer that, overall, Flight3 is not a very discriminative feature. Most Di-Graphs have very high mean Dist $_{B}$ values, which implies that this will not be helpful in separation of the users based on their keystroke data.

\subsection{Flight4}

Description: For a Di-graph, Flight4 is the time duration between press of the first key and release of the second key.

Inference: Table 9 presents the mean Dist $_{B}$ values for Flight 4 on all three devices. These values are similar to those of all other Flight features that we have analyzed so far. The mean Dist $_{B}$ values for Flight4 are better than those of KeyHold but not better than those of Flight1, Flight2, and Flight3. On the desktop, we find that most mean Dist $_{B}$ values are in the range of 0.4 to 0.7 , 


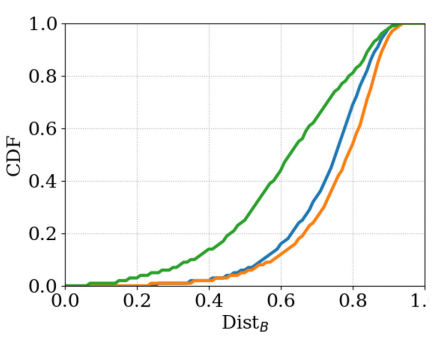

(a) KeyHold

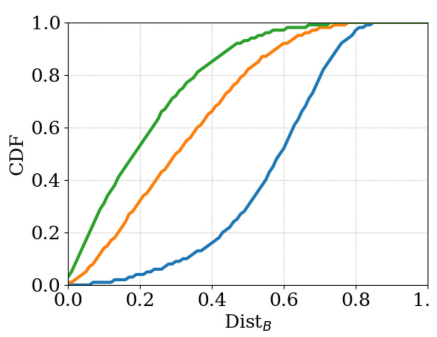

(d) Flight3

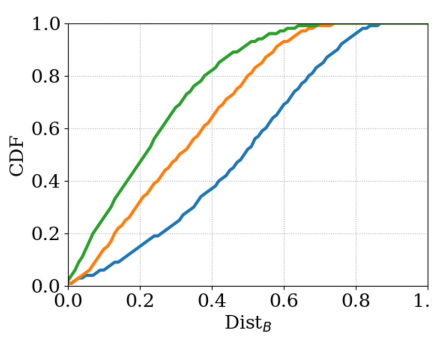

(b) Flight1

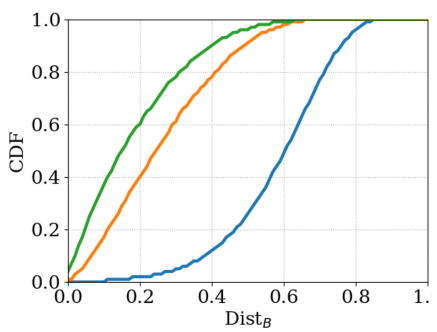

(e) Flight4

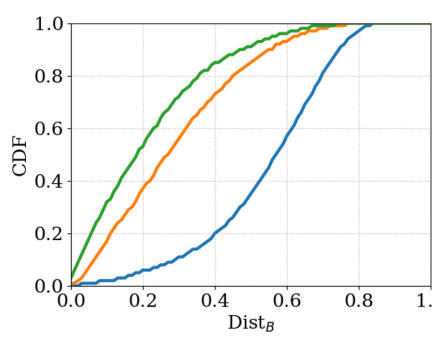

(c) Flight2

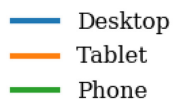

(f) Legend

Fig. 4. Comparing the Bhattacharyya distances of PDFs for all conventional features on desktop, tablet, and phone.

the least being $($ space,, $\mathrm{w})=0.4$ and highest being $(\mathrm{e}, \mathrm{space})=0.74$. On the tablet and on the phone, we see a few Di-Graphs having low mean Dist $_{B}$ values, but these are very few in number: (space: a,i,s,t) on tablet and (space: a,i,s,t) (e,n) on phone. The lowest and highest mean Dist $_{B}$ values on tablet are for $($ space,, $\mathrm{i})=0.098$ and $(\mathrm{s}, \mathrm{e})=0.46$, respectively; while on phone, they are $($ space, $\mathrm{i})=$ 0.06 and $(\mathrm{s}, \mathrm{e})=0.342$, respectively. It is also interesting to observe that the Di-graphs with lowest and highest mean Dist $_{B}$ values are for the same set of Di-Graphs on both the hand-held devices.

We illustrate the discriminability of the conventional features using CDFs of Dist $t_{B}$, shown in Figure 4. The CDFs help us compare the Bhattacharyya distances of each conventional feature among devices. Figure 4(a) shows that KeyHold value is not a discriminable feature on any of the devices. Though it has a slightly better curve on phone, it is still inefficient as a feature. From Figure 4(a), we can estimate that less than $10 \%$ of the test samples have low (hence desirable) values of $D_{i s t}$ of 0.2 or less. Around $60 \%$ of the test samples have Dist $t_{B}$ greater than 0.75 on desktop and tablet, and 0.5 on phone. These imply a very high overlap of the PDFs for this feature, which makes it a weak feature.

For the features Flight1, Flight2, Flight3, and Flight4, Figures 4(b) through 4(e) show that these features have similar distribution of Dist $_{B}$ values with minor variations. A clear observation is that, though these features are clearly better than the KeyHold feature, they have poor discriminability on the desktop data. Flight1 has some noticeable improvement with about $60 \%$ of them having Dist $_{B}$ less than 0.5, while Flight2, Flight3, and Flight4 offer negligible improvements by having $40 \%$ with less than 0.5 for Dist $_{B}$.

For tablet and phone, there is improvement in discriminability on all the features; both have much better discriminability when compared to the desktop. We observe that the features seem to be slightly more discriminative on phone compared to the tablet. In Flight1 (Figure 4(b)), we see about $60 \%$ of the test samples have Dist $_{B}$ less than 0.4 on tablet and less than 0.3 on phone. In the case of Flight2 (Figure 4(c)), we see about $60 \%$ of the test samples have Dist $t_{B}$ less than 0.3 for tablet and less than 0.25 for phone. With Flight 3 (Figure $4(\mathrm{~d})$ ), we see about $60 \%$ of them have Dist ${ }_{B}$ 
less than 0.4 for tablet and less than 0.25 for phone, which is very similar to Flight2. Last, Flight4 (Figure $4(\mathrm{e})$ ) shows $60 \%$ of Dist $_{B}$ values are less than 0.3 for tablet and less than 0.2 for phone.

\section{INFERENCE-CONVENTIONAL FEATURES}

Though the Flight features have slightly better Dist $_{B}$ values than KeyHold, none of these conventional features have high discriminative power that can be used to separate user keystroke data from each other to a high accuracy. We also observe that these conventional features aggregate all the values from the features without considering context, which might not be optimal, as variations in a feature value may occur due to the context of their appearance. We therefore propose a set of context-sensitive features that try and take advantage of known information, like the language being typed and the words of that language.

\section{ANALYSIS OF PROPOSED CONTEXT-SENSITIVE FEATURES}

The proposed features are mentioned in Table 3. These features are extracted for each occurrence of a word as opposed to each occurrence of the Di-graph. We extract "WordHold": the time taken to type an entire word (from first press to last release). "AvgKeyHold": the average of all KeyHold values in the word. "AvgFlight1," "AvgFlight2," "AvgFlight3," "AvgFlight4": the average of the respective flight values for Di-graphs in the word. "StdKeyHold": the standard deviation of all KeyHold values in the word. "StdFlight1," "StdFlight2," "StdFlight3," "StdFlight4": the standard deviation of the respective flight values for Di-graphs in the word.

We analyze the discriminability of our proposed features by using the same methods that were used to analyze the conventional features. We selected 20 of the highest occurring words in our dataset and generated the PDFs for each proposed feature for every user on all three devices. We then computed the Dist $_{B}$ values for the corresponding PDFs for every pair of users.

\subsection{WordHold}

Description: WordHold is the time duration between the first key pressed to the last key released in a word. We only consider the sequence of keystrokes as forming a word if it is done without any deviations, such as backspaces or delete keys being pressed.

Inference: Table 10 presents the mean Dist $_{B}$ values for WordHold on all three devices. The mean values of Dist $_{B}$ for WordHold are considerably better than those of all the conventional features that were analyzed in the previous section on corresponding devices. Most mean Dist $_{B}$ values for desktop are low and lie around 0.1 to 0.3 , implying much less overlap among the PDFs, the highest mean $\operatorname{Dist}_{B}$ value being for "that" $=0.397$. For tablet, the mean Dist $_{B}$ values for all words were extremely low, which is very desirable; this implies that the feature is good for differentiating between the users. All values are below 0.25 and the majority of them are below 0.1 . The highest mean Dist $_{B}$ value was for "see" $=0.232$. We observe phone to be similar to tablet with all words having extremely low $\operatorname{Dist}_{B}$ values. The least and highest mean Dist $_{B}$ values are for "words" = 0.005 and "see" $=0.106$, respectively.

Overall, the discriminability that this feature offers is very high. All three devices have very low values for mean Dist $_{B}$ values. These values are especially low on hand-held devices, and this appears to be a very good feature for user separation tasks like identification.

\subsection{AvgFlight1}

Description: AvgFlight1 is the average of all Flight1 values occurring within the context of a word. Flight1 value of all Di-graphs in the word are summed and divided by the number of Di-graphs.

Inference: Table 11 presents the mean Dist $_{B}$ values for AvgFlight1 on all three devices. The mean values of Dist $_{B}$ for AvgFlight1 are better than those of all the conventional features and are 
Table 10. The Inter-User Dist ${ }_{B}$ Values for WordHold Distributions across All Devices

\begin{tabular}{|c|c|c|c|c|c|c|c|c|c|c|}
\hline Device & \multicolumn{3}{|c|}{ Desktop } & \multicolumn{3}{c|}{ Tablet } & \multicolumn{3}{c|}{ Phone } \\
\hline \hline Word & Mean & StD & Median & Mean & StD & Median & Mean & StD & Median \\
\hline are & 0.391 & 0.175 & 0.4 & 0.099 & 0.092 & 0.081 & 0.069 & 0.073 & 0.046 \\
\hline carefully & 0.072 & 0.064 & 0.074 & \multicolumn{3}{|c|}{-} & & 0.026 & 0.04 & 0 \\
\hline data & 0.305 & 0.135 & 0.323 & 0.012 & 0.027 & 0 & 0.034 & 0.05 & 0 \\
\hline different & 0.153 & 0.077 & 0.143 & 0.01 & 0.024 & 0 & \multicolumn{3}{c|}{-} \\
\hline first & 0.288 & 0.085 & 0.29 & 0.02 & 0.037 & 0 & \multicolumn{3}{c|}{-} \\
\hline have & 0.221 & 0.182 & 0.239 & 0.028 & 0.057 & 0 & 0.024 & 0.042 & 0 \\
\hline lines & 0.238 & 0.151 & 0.273 & 0.03 & 0.048 & 0 & 0.038 & 0.09 & 0 \\
\hline not & 0.186 & 0.163 & 0.158 & 0.058 & 0.074 & 0.026 & 0.036 & 0.053 & 0 \\
\hline overlap & 0.091 & 0.036 & 0.069 & 0.012 & 0.028 & 0 & & - & \\
\hline phase & 0.167 & 0.075 & 0.185 & 0.128 & 0.148 & 0.069 & 0.031 & 0.048 & 0 \\
\hline see & 0.344 & 0.138 & 0.353 & 0.232 & 0.14 & 0.299 & 0.106 & 0.058 & 0.117 \\
\hline that & 0.397 & 0.168 & 0.443 & 0.04 & 0.063 & 0 & 0.041 & 0.056 & 0 \\
\hline the & 0.52 & 0.14 & 0.538 & 0.074 & 0.081 & 0.057 & 0.042 & 0.07 & 0 \\
\hline there & 0.221 & 0.09 & 0.237 & 0.156 & 0.139 & 0.069 & 0.041 & 0.087 & 0 \\
\hline this & 0.235 & 0.142 & 0.221 & 0.058 & 0.069 & 0.048 & 0.045 & 0.057 & 0.032 \\
\hline two & 0.179 & 0.148 & 0.154 & 0.034 & 0.035 & 0.033 & 0.019 & 0.03 & 0 \\
\hline type & 0.119 & 0.155 & 0.035 & 0.035 & 0.054 & 0 & & - & \\
\hline will & 0.193 & 0.153 & 0.192 & 0.035 & 0.046 & 0 & 0.013 & 0.027 & 0 \\
\hline with & 0.173 & 0.146 & 0.139 & 0.025 & 0.041 & 0 & 0.022 & 0.035 & 0 \\
\hline words & 0.133 & 0.104 & 0.121 & 0.069 & 0.107 & 0 & 0.005 & 0.018 & 0 \\
\hline
\end{tabular}

comparable to those of WordHold on corresponding devices. Most mean Dist $_{B}$ values for desktop are low and lie around 0.1 to 0.25 , implying less overlap among the PDFs. This feature is more discriminable than the WordHold feature on desktop. For tablet again, the mean Dist $_{B}$ values for all words were extremely low, which is very desirable. All values are below 0.25 and the majority of them are below 0.1 . We observe phone to be similar to tablet with all words having extremely low Dist $_{B}$ values. Overall, the discriminability that this feature offers is also very high. All three devices have very low values for mean $\operatorname{Dist}_{B}$ values. These values are especially low on hand-held devices.

\subsection{AvgFlight2}

Description: AvgFlight2 is the average of all Flight2 values occurring within the context of a word. Flight2 value of all Di-graphs in the word are summed and divided by the number of Di-graphs.

Inference: Table 12 presents the mean Dist $_{B}$ values for AvgFlight 2 on all three devices. The mean values of Dist $_{B}$ for AvgFlight2 are better than those of all the conventional features and are comparable to those of WordHold and AvgFlight1 on corresponding devices. Most mean Dist $_{B}$ values for desktop are low and lie around 0.1 to 0.4. The highest mean Dist $_{B}$ value was for "the" = 0.562 , which seems to be a rare case, as most values are lesser than 0.3. Again, in the case of hand-held devices, we observe very low mean Dist $_{B}$ values. For tablets, the mean Dist $_{B}$ values for all words were extremely low, which is very desirable; this implies that the feature is good for differentiating between the users. All values are below 0.27 and the majority of them lie below 0.1 . It is intriguing that the word "that" had mean Dist $_{B}$ value of $0, \mathrm{StD}=0$ and median $=0$, meaning no overlaps between any two user PDFs, theoretically providing $100 \%$ separation. We observe phone 
Table 11. The Inter-User Dist ${ }_{B}$ for AvgFlight1 Distributions across All Devices

\begin{tabular}{|c|c|c|c|c|c|c|c|c|c|c|}
\hline Device & \multicolumn{3}{|c|}{ Desktop } & \multicolumn{3}{c|}{ Tablet } & \multicolumn{3}{c|}{ Phone } \\
\hline \hline Word & Mean & StD & Median & Mean & StD & Median & Mean & StD & Median \\
\hline are & 0.236 & 0.216 & 0.18 & 0.14 & 0.104 & 0.122 & 0.152 & 0.105 & 0.127 \\
\hline carefully & 0.024 & 0.037 & 0 & \multicolumn{3}{|c|}{-} & & 0.128 & 0.04 & 0.154 \\
\hline data & 0.182 & 0.222 & 0.071 & 0.061 & 0.053 & 0.069 & 0.074 & 0.088 & 0.061 \\
\hline different & 0.186 & 0.089 & 0.244 & 0.058 & 0.087 & 0 & \multicolumn{3}{c|}{-} \\
\hline first & 0.181 & 0.1 & 0.148 & 0.039 & 0.061 & 0 & \multicolumn{3}{c|}{-} \\
\hline have & 0.153 & 0.192 & 0.078 & 0.067 & 0.071 & 0.062 & 0.09 & 0.106 & 0.069 \\
\hline lines & 0.122 & 0.153 & 0.036 & 0.077 & 0.064 & 0.069 & 0.15 & 0.105 & 0.143 \\
\hline not & 0.094 & 0.131 & 0 & 0.094 & 0.087 & 0.075 & 0.043 & 0.054 & 0 \\
\hline overlap & 0.046 & 0.036 & 0.069 & 0.037 & 0.059 & 0 & & - & \\
\hline phase & 0.186 & 0.146 & 0.215 & 0.069 & 0.062 & 0.069 & 0.205 & 0.094 & 0.196 \\
\hline see & 0.277 & 0.216 & 0.267 & 0.205 & 0.067 & 0.215 & 0.102 & 0.104 & 0.067 \\
\hline that & 0.165 & 0.152 & 0.105 & 0.088 & 0.091 & 0.065 & 0.085 & 0.094 & 0.067 \\
\hline the & 0.403 & 0.231 & 0.442 & 0.123 & 0.102 & 0.104 & 0.098 & 0.116 & 0.06 \\
\hline there & 0.101 & 0.205 & 0 & 0.056 & 0.086 & 0 & 0.102 & 0.151 & 0 \\
\hline this & 0.177 & 0.148 & 0.176 & 0.113 & 0.085 & 0.101 & 0.126 & 0.107 & 0.114 \\
\hline two & 0.131 & 0.105 & 0.101 & 0.045 & 0.052 & 0.033 & 0.018 & 0.04 & 0 \\
\hline type & 0.192 & 0.215 & 0.17 & 0.08 & 0.061 & 0.101 & \multicolumn{3}{|c|}{-} \\
\hline will & 0.095 & 0.16 & 0.033 & 0.058 & 0.079 & 0 & 0.072 & 0.086 & 0.033 \\
\hline with & 0.151 & 0.136 & 0.125 & 0.061 & 0.074 & 0.067 & 0.073 & 0.081 & 0.038 \\
\hline words & 0.117 & 0.135 & 0.077 & 0.046 & 0.036 & 0.069 & 0.059 & 0.066 & 0.065 \\
\hline
\end{tabular}

to be similar to tablet with all words having extremely low Dist $_{B}$ values. Even the highest value is for the word "carefully" $=0.164$, which is low.

We infer that the Discriminability offered by this feature is high. All three devices have very low values for mean Dist $_{B}$, especially extremely low values on hand-held devices. It appears to be a very good feature for authentication/verification purposes.

\subsection{AvgFlight3}

Description: AvgFlight3 is the average of all Flight3 values occurring within the context of a word. Flight3 value of all Di-graphs in the word are summed and divided by the number of Di-graphs.

Inference: Table 13 presents the mean Dist $_{B}$ values for AvgFlight3 on all three devices. The mean values of Dist $_{B}$ for AvgFlight3 are better than those of all the conventional features and are comparable to those of WordHold, AvgFlight1, and AvgFlight2 on corresponding devices. Most mean $\operatorname{Dist}_{B}$ values for desktop are low and lie around 0.1 to 0.4 . The highest mean Dist $_{B}$ value was for the word "the" $=0.504$, which is among the very few words with Dist $_{B}$ greater than 0.4 for this feature. For tablets, the mean Dist $_{B}$ values for all words were extremely low. All values lie below 0.26 except for "see" and the majority of them lie below 0.1 . Again, we observe phone to be similar to tablet with all words having extremely low Dist $_{B}$ values of less than 0.2 .

Overall, this feature offers high discriminability. All three devices have very low values for mean $\operatorname{Dist}_{B}$. Once again, the values on hand-held devices are much lower than those of the desktop.

\subsection{AvgFlight4}

Description: AvgFlight 4 is the average of all Flight 4 values occurring within the context of a word. Flight3 value of all Di-graphs in the word are summed and divided by the number of Di-graphs. 
Table 12. The Inter-User Dist $_{b}$ Values for AvgFlight2 Distributions across All Devices

\begin{tabular}{|c|l|c|c|c|c|c|c|c|c|c|}
\hline Device & \multicolumn{3}{|c|}{ Desktop } & \multicolumn{3}{c|}{ Tablet } & \multicolumn{3}{c|}{ Phone } \\
\hline \hline Word & Mean & StD & Median & Mean & StD & Median & Mean & StD & Median \\
\hline are & 0.415 & 0.21 & 0.449 & 0.159 & 0.117 & 0.155 & 0.126 & 0.105 & 0.109 \\
\hline carefully & 0.083 & 0.014 & 0.074 & \multicolumn{3}{|c|}{-} & & 0.164 & 0.071 & 0.186 \\
\hline data & 0.41 & 0.161 & 0.417 & 0.068 & 0.072 & 0.067 & 0.068 & 0.115 & 0 \\
\hline different & 0.123 & 0.045 & 0.124 & 0.075 & 0.119 & 0.03 & \multicolumn{3}{c|}{-} \\
\hline first & 0.28 & 0.152 & 0.237 & 0.063 & 0.086 & 0 & \multicolumn{3}{c|}{} \\
\hline have & 0.305 & 0.21 & 0.322 & 0.047 & 0.076 & 0 & 0.103 & 0.141 & 0 \\
\hline lines & 0.247 & 0.201 & 0.273 & 0.114 & 0.107 & 0.069 & 0.043 & 0.072 & 0 \\
\hline not & 0.173 & 0.172 & 0.148 & 0.083 & 0.091 & 0.066 & 0.044 & 0.068 & 0 \\
\hline overlap & 0.124 & 0.046 & 0.138 & 0.045 & 0.077 & 0 & & - & \\
\hline phase & 0.281 & 0.09 & 0.268 & 0.231 & 0.144 & 0.138 & 0.03 & 0.046 & 0 \\
\hline see & 0.34 & 0.184 & 0.341 & 0.261 & 0.128 & 0.285 & 0.119 & 0.105 & 0.114 \\
\hline that & 0.401 & 0.235 & 0.414 & 0 & 0 & 0 & 0.069 & 0.093 & 0.031 \\
\hline the & 0.562 & 0.157 & 0.595 & 0.116 & 0.102 & 0.104 & 0.089 & 0.116 & 0.046 \\
\hline there & 0.308 & 0.213 & 0.27 & 0.23 & 0.12 & 0.167 & 0.099 & 0.144 & 0 \\
\hline this & 0.261 & 0.168 & 0.238 & 0.128 & 0.096 & 0.118 & 0.109 & 0.107 & 0.085 \\
\hline two & 0.202 & 0.183 & 0.215 & 0.032 & 0.05 & 0 & 0.021 & 0.033 & 0 \\
\hline type & 0.182 & 0.152 & 0.118 & 0.076 & 0.066 & 0.082 & & - & \\
\hline will & 0.181 & 0.177 & 0.169 & 0.063 & 0.096 & 0 & 0.03 & 0.05 & 0 \\
\hline with & 0.184 & 0.158 & 0.151 & 0.063 & 0.057 & 0.071 & 0.08 & 0.092 & 0.035 \\
\hline words & 0.186 & 0.094 & 0.191 & 0.145 & 0.168 & 0.077 & 0.038 & 0.052 & 0 \\
\hline
\end{tabular}

Inference: Table 14 presents the mean Dist $_{B}$ values for AvgFlight 4 on all three devices. The mean values of Dist $_{B}$ for AvgFlight4 are better than those of all the conventional features and are comparable to those of WordHold, AvgFlight1, AvgFlight2, and AvgFlight3 on corresponding devices. For desktop, most mean Dist $_{B}$ values are low and lie around 0.1 to 0.4. For tablet and phone, the mean Dist $_{B}$ values for all words were extremely low, which is very desirable. For tablet, all values lie below 0.2 and the majority of them lie below 0.1 . For phone, all words have extremely low Dist $_{B}$ values of less than 0.17 . We again came across a peculiar case on phone, where for the word phase, theoretically, mean of $0, \mathrm{StD}=0$, and median $=0$ implies no overlap between PDFs of any two users.

We infer that the feature AvgFlight4 has high discriminability. All devices have very low values for mean Dist $_{B}$ and extremely low values on hand-held devices.

\subsection{AvgKeyHold}

Description: AvgKeyHold is the average of all KeyHold values occurring within the context of a word. KeyHold value of all Uni-graphs in the word are summed and divided by the number of Uni-graphs.

Inference: Table 15 presents the mean Dist $_{B}$ values for AvgKeyHold. The mean values of Dist $t_{B}$ for AvgKeyHold are better than those of all the conventional features but are inferior to those of WordHold, AvgFlight1, AvgFlight2, AvgFlight3, and AvgFlight4 on corresponding devices, except for desktop, where they are similar to them. Most mean Dist $_{B}$ values for desktop are low and lie around 0.1 to 0.3 , implying less overlap among the PDFs. The word "carefully" has a mean, std, and median Dist $_{B}$ value of 0 ; therefore, theoretically, this feature should provide $100 \%$ separation 
Table 13. The Inter-User Dist $_{B}$ Values for AvgFlight3 Distributions across All Devices

\begin{tabular}{|c|c|c|c|c|c|c|c|c|c|c|}
\hline Device & \multicolumn{3}{|c|}{ Desktop } & \multicolumn{3}{c|}{ Tablet } & \multicolumn{3}{c|}{ Phone } \\
\hline \hline Word & Mean & StD & Median & Mean & StD & Median & Mean & StD & Median \\
\hline are & 0.407 & 0.201 & 0.432 & 0.153 & 0.12 & 0.132 & 0.121 & 0.098 & 0.113 \\
\hline carefully & 0.057 & 0.089 & 0 & \multicolumn{3}{|c|}{-} & & 0.128 & 0.04 & 0.154 \\
\hline data & 0.476 & 0.127 & 0.483 & 0.054 & 0.061 & 0.035 & 0.051 & 0.089 & 0 \\
\hline different & 0.186 & 0.067 & 0.214 & 0.053 & 0.098 & 0 & \multicolumn{3}{c|}{-} \\
\hline first & 0.232 & 0.166 & 0.194 & 0.04 & 0.075 & 0 & \multicolumn{3}{c|}{-} \\
\hline have & 0.283 & 0.225 & 0.291 & 0.081 & 0.092 & 0.069 & 0.171 & 0.164 & 0.138 \\
\hline lines & 0.299 & 0.193 & 0.311 & 0.061 & 0.06 & 0.069 & 0.07 & 0.099 & 0.037 \\
\hline not & 0.174 & 0.189 & 0.089 & 0.109 & 0.105 & 0.081 & 0.049 & 0.064 & 0 \\
\hline overlap & 0.132 & 0.029 & 0.138 & 0.035 & 0.037 & 0.033 & & - \\
\hline phase & 0.219 & 0.112 & 0.225 & 0.171 & 0.169 & 0.138 & 0.132 & 0.055 & 0.126 \\
\hline see & 0.453 & 0.152 & 0.458 & 0.346 & 0.105 & 0.302 & 0.078 & 0.116 & 0.033 \\
\hline that & 0.304 & 0.196 & 0.371 & 0.097 & 0.104 & 0.065 & 0.075 & 0.093 & 0.033 \\
\hline the & 0.504 & 0.173 & 0.54 & 0.134 & 0.115 & 0.116 & 0.088 & 0.109 & 0.051 \\
\hline there & 0.281 & 0.145 & 0.324 & 0.253 & 0.111 & 0.237 & 0.089 & 0.15 & 0 \\
\hline this & 0.294 & 0.189 & 0.266 & 0.131 & 0.113 & 0.11 & 0.106 & 0.107 & 0.076 \\
\hline two & 0.249 & 0.199 & 0.225 & 0.011 & 0.026 & 0 & 0.032 & 0.043 & 0 \\
\hline type & 0.159 & 0.171 & 0.131 & 0.092 & 0.065 & 0.074 & \multicolumn{3}{|c|}{-1} \\
\hline will & 0.264 & 0.22 & 0.181 & 0.054 & 0.073 & 0 & 0.023 & 0.047 & 0 \\
\hline with & 0.192 & 0.169 & 0.173 & 0.056 & 0.064 & 0.061 & 0.123 & 0.15 & 0.077 \\
\hline words & 0.173 & 0.146 & 0.139 & 0.112 & 0.123 & 0.069 & 0.028 & 0.043 & 0 \\
\hline
\end{tabular}

among users. For tablet, the mean $\operatorname{Dist}_{B}$ values are low but are slightly higher than their corresponding values in WordHold and AvgFlight1 through AvgFlight4. All values lie below 0.32 and the majority of them are below 0.2 . The least and the highest mean Dist $_{B}$ value are for "there" = 0.075 and "are" $=0.31$, respectively. With phone, we make similar observations, as that of tablet Dist $_{B}$ values and all values are less than 0.39 . The least and highest values were for the words "not" $=0.138$ and "carefully" $=0.382$, respectively. Overall, AvgKeyHold is not as discriminable as WordHold, AvgFlight1, AvgFlight2, AvgFlight3, and AvgFlight4 and does not provide much improvement from the conventional features.

\subsection{StdFlight 1}

Description: StdFlight1 is the standard deviation of all Flight1 values occurring within the context of a word.

Inference: Table 16 presents the mean Dist $_{B}$ values for StdFlight 1 on all three devices. Similar to the AvgKeyHold, mean values of Dist $_{B}$ for Std-Word-Flight1 are better than those of all the conventional features but are inferior to those of WordHold, AvgFlight1, AvgFlight2, AvgFlight3, and AvgFlight4 on corresponding devices, except for desktop, where they are similar to them. Most mean Dist $_{B}$ values for desktop lie around 0.1 to 0.6 , implying considerable overlap among the PDFs. For tablet, the mean Dist $_{B}$ values are low but are slightly higher than their corresponding values in WordHold and AvgFlight1 through AvgFlight4. All values are below 0.30 except for "see," and the majority of them lie below 0.25 . With phone, we make similar observations as that of tablet Dist $_{B}$ values: All values are less than 0.25 . We can infer that StdFlight1 is not as discriminable as WordHold, AvgFlight1, AvgFlight2, AvgFlight3, and AvgFlight4. 
Table 14. The Inter-User Mean Dist $_{B}$ Values for AvgFlight4 Distributions across All Devices

\begin{tabular}{|c|c|c|c|c|c|c|c|c|c|c|}
\hline Device & \multicolumn{3}{|c|}{ Desktop } & \multicolumn{3}{c|}{ Tablet } & \multicolumn{3}{c|}{ Phone } \\
\hline \hline Word & Mean & StD & Median & Mean & StD & Median & Mean & StD & Median \\
\hline are & 0.354 & 0.169 & 0.367 & 0.127 & 0.099 & 0.106 & 0.11 & 0.104 & 0.09 \\
\hline carefully & 0.123 & 0.077 & 0.074 & \multicolumn{3}{|c|}{-} & & 0.166 & 0.036 & 0.154 \\
\hline data & 0.18 & 0.186 & 0.128 & 0.037 & 0.059 & 0 & 0.064 & 0.09 & 0 \\
\hline different & 0.024 & 0.037 & 0 & 0.111 & 0.125 & 0.065 & \multicolumn{3}{c|}{-} \\
\hline first & 0.215 & 0.124 & 0.195 & 0.05 & 0.057 & 0 & \multicolumn{3}{c|}{-} \\
\hline have & 0.228 & 0.133 & 0.182 & 0.047 & 0.068 & 0 & 0.057 & 0.076 & 0 \\
\hline lines & 0.31 & 0.202 & 0.272 & 0.085 & 0.078 & 0.069 & 0.047 & 0.072 & 0 \\
\hline not & 0.213 & 0.161 & 0.203 & 0.076 & 0.075 & 0.068 & 0.045 & 0.067 & 0 \\
\hline overlap & 0.148 & 0.136 & 0.138 & 0.075 & 0.111 & 0 & & - & \\
\hline phase & 0.162 & 0.135 & 0.146 & 0.069 & 0.107 & 0 & 0 & 0 & 0 \\
\hline see & 0.289 & 0.157 & 0.297 & 0.19 & 0.119 & 0.178 & 0.073 & 0.073 & 0.078 \\
\hline that & 0.31 & 0.185 & 0.267 & 0.113 & 0.073 & 0.067 & 0.037 & 0.039 & 0.031 \\
\hline the & 0.506 & 0.139 & 0.512 & 0.099 & 0.091 & 0.072 & 0.069 & 0.099 & 0 \\
\hline there & 0.287 & 0.166 & 0.244 & 0.023 & 0.036 & 0 & 0.1 & 0.11 & 0.061 \\
\hline this & 0.322 & 0.117 & 0.331 & 0.098 & 0.076 & 0.098 & 0.089 & 0.091 & 0.061 \\
\hline two & 0.191 & 0.136 & 0.194 & 0.057 & 0.087 & 0 & 0.032 & 0.034 & 0.03 \\
\hline type & 0.269 & 0.176 & 0.281 & 0.069 & 0.085 & 0 & & - & \\
\hline will & 0.293 & 0.112 & 0.281 & 0.053 & 0.059 & 0.033 & 0.055 & 0.08 & 0 \\
\hline with & 0.212 & 0.132 & 0.202 & 0.073 & 0.061 & 0.074 & 0.097 & 0.093 & 0.076 \\
\hline words & 0.142 & 0.076 & 0.113 & 0.095 & 0.034 & 0.077 & 0.025 & 0.036 & 0 \\
\hline
\end{tabular}

\subsection{StdFlight2}

Description: StdFlight2 is the standard deviation of all Flight2 values occurring within the context of a word.

Inference: Table 17 presents the mean Dist $_{B}$ values for StdFlight 2 on all three devices. The mean values of Dist $_{B}$ for Std-Word-Flight2 are better than those of all the conventional features but are inferior to those of WordHold, AvgFlight1, AvgFlight2, AvgFlight3, and AvgFlight4 on corresponding devices, but for desktop they are similar to them. Most mean Dist $_{B}$ values for desktop lie around 0.1 to 0.48 , implying considerable overlap among the PDFs. For tablet, the mean Dist $_{B}$ values are low but are slightly higher than their corresponding values in WordHold and AvgFlight1 through AvgFlightt4. All values are below 0.26, and the majority of them lie below 0.2 . With phone, we make similar observations as that of tablet Dist $_{B}$ values, all of which are less than 0.24 . Discriminability is not as high as WordHold, AvgFlight1, AvgFlight2, AvgFlight3, and AvgFlight4 using this feature. This feature also does not provide an improvement over conventional features.

\subsection{StdFlight3}

Description: StdFlight3 is the standard deviation of all Flight3 values occurring within the context of a word.

Inference: Table 18 presents the mean Dist $_{B}$ values for StdFlight 3 on all three devices. The mean values of Dist $_{B}$ for Std-Word-Flight3 are better than those of all the conventional features but are inferior to those of WordHold, AvgFlight1, AvgFlight2, AvgFlight3, and AvgFlight4 on corresponding devices, but again for desktop they are similar. Most mean Dist $_{B}$ values for desktop lie around 0.1 to 0.4 , implying considerable overlap among the PDFs. For tablet, the mean Dist $_{B}$ 
Table 15. The Inter-User Dist ${ }_{B}$ Values for AvgKeyHold Distributions across All Devices

\begin{tabular}{|c|c|c|c|c|c|c|c|c|c|c|}
\hline Device & \multicolumn{3}{|c|}{ Desktop } & \multicolumn{3}{c|}{ Tablet } & \multicolumn{3}{c|}{ Phone } \\
\hline \hline Word & Mean & StD & Median & Mean & StD & Median & Mean & StD & Median \\
\hline are & 0.281 & 0.247 & 0.233 & 0.31 & 0.17 & 0.316 & 0.25 & 0.182 & 0.247 \\
\hline carefully & 0 & 0 & 0 & \multicolumn{3}{|c|}{-} & & 0.382 & 0.209 & 0.371 \\
\hline data & 0.143 & 0.159 & 0.128 & 0.145 & 0.155 & 0.096 & 0.259 & 0.153 & 0.242 \\
\hline different & 0.234 & 0.179 & 0.172 & 0.181 & 0.21 & 0.101 & \multicolumn{3}{c|}{-} \\
\hline first & 0.252 & 0.177 & 0.253 & 0.264 & 0.189 & 0.253 & \multicolumn{3}{c|}{-} \\
\hline have & 0.231 & 0.192 & 0.176 & 0.182 & 0.17 & 0.139 & 0.198 & 0.146 & 0.211 \\
\hline lines & 0.203 & 0.217 & 0.139 & 0.233 & 0.11 & 0.265 & 0.164 & 0.172 & 0.074 \\
\hline not & 0.238 & 0.172 & 0.214 & 0.291 & 0.178 & 0.296 & 0.138 & 0.153 & 0.072 \\
\hline overlap & 0.208 & 0.116 & 0.228 & 0.136 & 0.116 & 0.151 & & - & \\
\hline phase & 0.224 & 0.197 & 0.283 & 0.209 & 0.167 & 0.264 & 0.179 & 0.23 & 0.063 \\
\hline see & 0.3 & 0.223 & 0.296 & 0.255 & 0.132 & 0.273 & 0.207 & 0.081 & 0.209 \\
\hline that & 0.218 & 0.272 & 0.074 & 0.167 & 0.079 & 0.207 & 0.212 & 0.091 & 0.229 \\
\hline the & 0.44 & 0.243 & 0.439 & 0.244 & 0.161 & 0.251 & 0.242 & 0.179 & 0.242 \\
\hline there & 0.118 & 0.192 & 0.044 & 0.075 & 0.069 & 0.069 & 0.359 & 0.169 & 0.409 \\
\hline this & 0.29 & 0.195 & 0.282 & 0.29 & 0.159 & 0.292 & 0.256 & 0.182 & 0.262 \\
\hline two & 0.264 & 0.308 & 0.077 & 0.122 & 0.137 & 0.067 & 0.198 & 0.083 & 0.217 \\
\hline type & 0.159 & 0.15 & 0.135 & 0.166 & 0.084 & 0.162 & \multicolumn{3}{|c|}{-} \\
\hline will & 0.248 & 0.238 & 0.211 & 0.161 & 0.111 & 0.143 & 0.268 & 0.137 & 0.27 \\
\hline with & 0.277 & 0.218 & 0.259 & 0.165 & 0.172 & 0.105 & 0.272 & 0.095 & 0.242 \\
\hline words & 0.146 & 0.134 & 0.126 & 0.026 & 0.04 & 0 & 0.228 & 0.186 & 0.2 \\
\hline
\end{tabular}

values are low but are slightly higher than their corresponding values in WordHold and AvgFlight1 through AvgFlight4. All values lie below 0.35, and the majority of them are below 0.3, The least and the highest mean Dist $_{B}$ values were for "will" $=0.061$ and "see" $=0.322$, respectively. The values for mean Dist $_{B}$ are also low for phone. All values lie below 0.24 The least mean Dist $_{B}$ value was for "words" $=0.078$ and highest for "the" $=0.236$. Overall, discriminability is not as high as WordHold, AvgFlight1, AvgFlight2, AvgFlight3, and AvgFlight4 using this feature. It does not provide desirable improvement from the conventional features.

\subsection{StdFlight4}

Description: StdFlight4 is the standard deviation of all Flight4 values occurring within the context of a word.

Inference: Table 19 presents the mean Dist $_{B}$ values for StdFlight 4 . The mean values of Dist $t_{B}$ for Std-Word-Flight4 are also better than those of all the conventional features but are inferior to those of WordHold, AvgFlight1, AvgFlight2, AvgFlight3, and AvgFlight4 on corresponding devices, but again for desktop they are similar. Most mean Dist $_{B}$ values for desktop lie around 0.1 to 0.5 , implying considerable overlap among the PDFs. The least and the highest mean Dist $_{B}$ values are for "carefully" $=0.025$ and "the" $=0.67$, respectively. For tablet the mean Dist $_{B}$ values are low, but are slightly higher than their corresponding values in WordHold, AvgFlight1 through AvgFlight4, all values being below 0.35 , and the majority of them lying below 0.3 , least value for "data" $=0.05$ and highest for "are" $=0.285$. With phone, we make similar observations as that of tablet Dist $_{B}$ values. All Dist $_{B}$ values are less than 0.28 . The least and highest mean values are for "words" = 0.085 and "the" $=0.27$, respectively. We infer that StdFlight4 is not as discriminable as WordHold, 
Table 16. The Inter-User Dist $_{B}$ Values for StdFlight 1 Distributions across All Devices

\begin{tabular}{|c|c|c|c|c|c|c|c|c|c|c|}
\hline Device & \multicolumn{3}{|c|}{ Desktop } & \multicolumn{3}{c|}{ Tablet } & \multicolumn{3}{c|}{ Phone } \\
\hline \hline Word & Mean & StD & Median & Mean & StD & Median & Mean & StD & Median \\
\hline are & 0.511 & 0.217 & 0.531 & 0.3 & 0.127 & 0.297 & 0.205 & 0.112 & 0.213 \\
\hline carefully & 0.049 & 0.038 & 0.074 & \multicolumn{3}{|c|}{-} & & 0.179 & 0.04 & 0.154 \\
\hline data & 0.195 & 0.187 & 0.101 & 0.095 & 0.059 & 0.091 & 0.126 & 0.136 & 0.077 \\
\hline different & 0.143 & 0 & 0.143 & 0.134 & 0.114 & 0.154 & \multicolumn{3}{c|}{-} \\
\hline first & 0.171 & 0.121 & 0.164 & 0.094 & 0.103 & 0.074 & \multicolumn{3}{c|}{-} \\
\hline have & 0.288 & 0.187 & 0.312 & 0.115 & 0.108 & 0.072 & 0.129 & 0.088 & 0.113 \\
\hline lines & 0.112 & 0.12 & 0.073 & 0.13 & 0.103 & 0.139 & 0.197 & 0.074 & 0.199 \\
\hline not & 0.405 & 0.289 & 0.475 & 0.229 & 0.137 & 0.215 & 0.138 & 0.116 & 0.108 \\
\hline overlap & 0.023 & 0.036 & 0 & 0.024 & 0.035 & 0 & & - & \\
\hline phase & 0.059 & 0.065 & 0.068 & 0.218 & 0.073 & 0.207 & 0.232 & 0.175 & 0.148 \\
\hline see & 0.448 & 0.203 & 0.499 & 0.465 & 0.154 & 0.476 & 0.226 & 0.089 & 0.199 \\
\hline that & 0.268 & 0.123 & 0.257 & 0.268 & 0.124 & 0.194 & 0.147 & 0.123 & 0.146 \\
\hline the & 0.585 & 0.17 & 0.615 & 0.233 & 0.143 & 0.22 & 0.227 & 0.127 & 0.224 \\
\hline there & 0.168 & 0.161 & 0.148 & 0.069 & 0.062 & 0.069 & 0.172 & 0.113 & 0.198 \\
\hline this & 0.332 & 0.142 & 0.367 & 0.186 & 0.109 & 0.177 & 0.198 & 0.126 & 0.184 \\
\hline two & 0.349 & 0.175 & 0.414 & 0.183 & 0.092 & 0.201 & 0.116 & 0.092 & 0.088 \\
\hline type & 0.177 & 0.101 & 0.175 & 0.075 & 0.067 & 0.07 & & - & \\
\hline will & 0.146 & 0.118 & 0.152 & 0.065 & 0.07 & 0.072 & 0.179 & 0.138 & 0.154 \\
\hline with & 0.158 & 0.127 & 0.137 & 0.095 & 0.08 & 0.074 & 0.118 & 0.145 & 0.087 \\
\hline words & 0.052 & 0.057 & 0.036 & 0.023 & 0.036 & 0 & 0.082 & 0.076 & 0.072 \\
\hline
\end{tabular}

AvgFlight1, AvgFlight2, AvgFlight3 and AvgFlight4. This feature does not provide improvement over the conventional features.

\subsection{StdKeyHold}

Description: StdKeyHold is the standard deviation of all KeyHold values occurring within the context of a word.

Inference: Table 20 presents the mean Dist $_{B}$ values for StdKeyHold on the three devices. The mean values of Dist $_{B}$ for StdKeyHold are similar to those of all the conventional features and are much inferior to those of all other proposed features on corresponding devices, but again for desktop they are similar to conventional feature values. Most mean Dist $_{B}$ values for desktop lie around 0.2 to 0.5 , which implies lesser separability. For tablet, the mean Dist $_{B}$ values are high, all values are above 0.26 , and the majority of them lie above 0.4 . The least mean $D_{i s} t_{B}$ value was for "phase" = 0.26 and highest for "first" $=0.517$. This implies that this feature offers very less discriminability. With phone, we make similar observations as that of tablet Dist $_{B}$ values: All values lie above 0.24 . The least mean Dist $_{B}$ value is for "carefully" $=0.245$. Overall, discriminability is low using this feature. All values on all devices suggest high overlaps in the PDFs; users are least separable using this among all the proposed features. Very few values on any device are desirable, and the majority are high; therefore, this feature is not a good feature for authentication/verification purposes.

We illustrate the discriminability of our proposed features using CDFs of Dist $_{B}$ values, as shown in Figure 5. The CDFs help us compare the separability of users by each feature on all devices. These are the observations for each feature in comparison to conventional features and other proposed features. Figure 5(a) shows the CDF for WordHold. It appears to have higher discriminability in desktop, about $60 \%$ of the samples having less than 0.4 Dist $_{B}$ and about $90 \%$ of them being less than 
Table 17. The Inter-User Dist $_{B}$ Values for StdFlight2 Distributions across All Devices

\begin{tabular}{|c|c|c|c|c|c|c|c|c|c|c|}
\hline Device & \multicolumn{3}{|c|}{ Desktop } & \multicolumn{3}{c|}{ Tablet } & \multicolumn{3}{c|}{ Phone } \\
\hline \hline Word & Mean & StD & Median & Mean & StD & Median & Mean & StD & Median \\
\hline are & 0.454 & 0.187 & 0.471 & 0.258 & 0.11 & 0.26 & 0.23 & 0.128 & 0.218 \\
\hline carefully & 0.049 & 0.077 & 0 & \multicolumn{3}{|c|}{-} & & 0.051 & 0.04 & 0.077 \\
\hline data & 0.195 & 0.163 & 0.161 & 0.102 & 0.172 & 0 & 0.145 & 0.134 & 0.154 \\
\hline different & 0.095 & 0.037 & 0.071 & 0.155 & 0.094 & 0.201 & \multicolumn{3}{c|}{-} \\
\hline first & 0.221 & 0.127 & 0.181 & 0.09 & 0.105 & 0.064 & \multicolumn{4}{c|}{} \\
\hline have & 0.184 & 0.141 & 0.181 & 0.13 & 0.081 & 0.129 & 0.117 & 0.102 & 0.072 \\
\hline lines & 0.115 & 0.123 & 0.088 & 0.093 & 0.079 & 0.077 & 0.133 & 0.096 & 0.129 \\
\hline not & 0.362 & 0.249 & 0.358 & 0.189 & 0.121 & 0.171 & 0.125 & 0.101 & 0.113 \\
\hline overlap & 0.045 & 0.035 & 0.067 & 0.067 & 0.06 & 0.07 & \multicolumn{3}{|c|}{-} \\
\hline phase & 0.049 & 0.056 & 0.033 & 0.158 & 0.074 & 0.167 & 0.223 & 0.082 & 0.222 \\
\hline see & 0.475 & 0.218 & 0.507 & 0.242 & 0.084 & 0.22 & 0.115 & 0.089 & 0.105 \\
\hline that & 0.288 & 0.106 & 0.253 & 0.118 & 0.085 & 0.065 & 0.15 & 0.119 & 0.161 \\
\hline the & 0.608 & 0.14 & 0.624 & 0.23 & 0.125 & 0.228 & 0.215 & 0.14 & 0.195 \\
\hline there & 0.213 & 0.081 & 0.231 & 0.164 & 0.082 & 0.139 & 0.199 & 0.121 & 0.189 \\
\hline this & 0.297 & 0.113 & 0.28 & 0.179 & 0.098 & 0.176 & 0.186 & 0.115 & 0.184 \\
\hline two & 0.33 & 0.155 & 0.327 & 0.074 & 0.051 & 0.069 & 0.084 & 0.124 & 0 \\
\hline type & 0.144 & 0.107 & 0.139 & 0.063 & 0.057 & 0.07 & \multicolumn{3}{|c|}{-} \\
\hline will & 0.215 & 0.108 & 0.23 & 0.085 & 0.08 & 0.069 & 0.159 & 0.17 & 0.072 \\
\hline with & 0.202 & 0.113 & 0.196 & 0.115 & 0.091 & 0.134 & 0.125 & 0.13 & 0.086 \\
\hline words & 0.1 & 0.091 & 0.088 & 0.118 & 0.032 & 0.139 & 0.117 & 0.108 & 0.077 \\
\hline
\end{tabular}

0.6 Dist $_{B}$. On phone and tablet, this feature is very good, as almost $50 \%$ and $40 \%$ samples have 0 Dist $_{B}$ on phone and tablet, respectively. $100 \%$ of the samples have below 0.35 Dist $_{B}$, which implies very good separation. We consider this feature to be evaluated in the next phase. Figure 5(b) shows the CDF for AvgFlight1. This shows an improved discriminability in desktop: $60 \%$ of the samples have less than 0.3 Dist $_{B}$ and around $25 \%$ at 0 . We also observe that Dist $_{B}$ of 0.6 covers $90 \%$ of the samples. On phone and tablet, this feature appears to be very good, as almost $35 \%$ and $25 \%$ of the samples have 0 Dist $_{B}$ on phone and tablet, respectively, and $100 \%$ below 0.4 , which implies very good separation. This feature is considered for evaluation in the next phase.

Figure 5(c) shows the CDF for AvgFlight2. We observe that it is slightly less discriminative than WordHold in desktop: Approximately $50 \%$ of the samples have less than 0.4 Dist $_{B}$ and $80 \%$ have less than 0.6. On phone and tablet, this feature is very good, as almost $40 \%$ and $30 \%$ of samples have $0 \mathrm{Dist}_{B}$ on phone and tablet, respectively. Approximately $100 \%$ are below $0.4 \mathrm{Dist}_{B}$, which implies very good separation. We considered this feature for evaluation in the next phase. Figure 5(d) shows the CDF for AvgFlight3. Again, this feature is slightly less discriminative than WordHold in desktop: Approximately $50 \%$ of all samples have less than 0.4 Dist $_{B}$ and about $80 \%$ have less than 0.6 . On phone and tablet, this feature is very good, as almost $40 \%$ and $30 \%$ of samples have 0 Dist $_{B}$ on phone and tablet, respectively. Approximately $100 \%$ are below 0.4 Dist $_{B}$, which implies very good separation. Feature is considered for evaluation in the next phase. Figure 5(e) shows that the CDF for AvgFlight4 is slightly more discriminative than AvgFlight3 with approximately $60 \%$ samples having less than 0.4 Dist $_{B}$ and $80 \%$ less than 0.6. On phone and tablet, this feature has almost $40 \%$ and $30 \%$ of samples with 0 Dist $_{B}$ on phone and tablet, respectively, and $100 \%$ below 0.4 Dist $_{B}$, which implies very good separation. We will be considering this feature for evaluation in the next phase. Figure 5(f) shows the CDF for StdFlight1. For desktop, about $60 \%$ of samples have 
Table 18. The Inter-User Dist ${ }_{B}$ Values for StdFlight3 Distributions across All Devices

\begin{tabular}{|c|l|c|c|c|c|c|c|c|c|c|}
\hline Device & \multicolumn{3}{|c|}{ Desktop } & \multicolumn{3}{c|}{ Tablet } & \multicolumn{3}{c|}{ Phone } \\
\hline \hline Word & Mean & StD & Median & Mean & StD & Median & Mean & StD & Median \\
\hline are & 0.59 & 0.135 & 0.577 & 0.287 & 0.115 & 0.287 & 0.194 & 0.101 & 0.193 \\
\hline carefully & 0.074 & 0.066 & 0.074 & \multicolumn{3}{|c|}{-} & & 0.149 & 0.121 & 0.186 \\
\hline data & 0.282 & 0.141 & 0.295 & 0.068 & 0.118 & 0 & 0.108 & 0.096 & 0.109 \\
\hline different & 0.071 & 0.064 & 0.071 & 0.185 & 0.087 & 0.164 & \multicolumn{3}{c|}{-} \\
\hline first & 0.221 & 0.055 & 0.216 & 0.104 & 0.078 & 0.081 & \multicolumn{4}{c|}{} \\
\hline have & 0.269 & 0.168 & 0.226 & 0.149 & 0.071 & 0.153 & 0.111 & 0.101 & 0.082 \\
\hline lines & 0.236 & 0.114 & 0.206 & 0.116 & 0.097 & 0.098 & 0.179 & 0.04 & 0.175 \\
\hline not & 0.373 & 0.174 & 0.356 & 0.213 & 0.121 & 0.214 & 0.109 & 0.089 & 0.109 \\
\hline overlap & 0.023 & 0.036 & 0 & 0.087 & 0.095 & 0.067 & & - & \\
\hline phase & 0.104 & 0.074 & 0.109 & 0.093 & 0.035 & 0.071 & 0.133 & 0.103 & 0.189 \\
\hline see & 0.544 & 0.104 & 0.554 & 0.322 & 0.095 & 0.297 & 0.157 & 0.105 & 0.155 \\
\hline that & 0.277 & 0.11 & 0.253 & 0.166 & 0.079 & 0.2 & 0.145 & 0.123 & 0.139 \\
\hline the & 0.613 & 0.138 & 0.633 & 0.285 & 0.123 & 0.283 & 0.236 & 0.118 & 0.218 \\
\hline there & 0.166 & 0.063 & 0.166 & 0.141 & 0.057 & 0.167 & 0.159 & 0.115 & 0.141 \\
\hline this & 0.371 & 0.123 & 0.384 & 0.191 & 0.11 & 0.182 & 0.22 & 0.128 & 0.217 \\
\hline two & 0.323 & 0.156 & 0.322 & 0.186 & 0.062 & 0.161 & 0.086 & 0.099 & 0.073 \\
\hline type & 0.08 & 0.048 & 0.07 & 0.127 & 0.052 & 0.121 & & - & \\
\hline will & 0.185 & 0.135 & 0.152 & 0.061 & 0.158 & 0 & 0.23 & 0.173 & 0.182 \\
\hline with & 0.198 & 0.115 & 0.173 & 0.136 & 0.123 & 0.092 & 0.104 & 0.094 & 0.077 \\
\hline words & 0.082 & 0.071 & 0.074 & 0.195 & 0.087 & 0.139 & 0.078 & 0.095 & 0.065 \\
\hline
\end{tabular}

less than 0.5 Dist $_{B}$ and $80 \%$ less than 0.7 , showing the feature is not very discriminative. On phone and tablet, this feature is better than desktop, but not better than the other features discussed for phone and desktop. About $60 \%$ of samples have below 0.3 and $90 \%$ have below 0.4 Dist $_{B}$, not as good as 5(a) to 5(e). We discard this proposed feature on grounds of not being discriminative enough. Figure $5(\mathrm{~g})$ shows the CDF for StdFlight2 very similar to StdFlight1; for desktop, about $60 \%$ of the values for Dist $_{B}$ are less than 0.5 and about $80 \%$ of them are less than 0.7 , implying that the feature is not very discriminative. On phone and tablet, this feature is better than desktop, but not better than the other features discussed for phone and desktop. About $60 \%$ of the samples have below 0.3 Dist $_{B}$ and $90 \%$ have below 0.4 , which is again not as good as $5($ a) to $5(\mathrm{e})$. We discard this feature, too, as not being discriminative enough. Figure 5(h) shows the CDF for StdFlight3. We observe that for desktop, $60 \%$ of the samples have less than 0.6 Dist $_{B}$ and $80 \%$ have less than 0.7 , which is not very discriminative. On phone and tablet, this feature is better than desktop, but they still have $60 \%$ of the Dist $_{B}$ values below 0.3 and $90 \%$ below 0.4 , which is not as good as $5($ a) to $5(\mathrm{e})$. We discard this feature, as it does not offer improvement over conventional features. Figure 5(i) shows the CDF for StdFlight4 very similar to StdFlight3. For desktop, $60 \%$ of values for Dist $_{B}$ are less than 0.6 Dist $_{B}$ and $80 \%$ less than 0.7 , not very discriminative. On phone and tablet, this feature is better than desktop, but not better than the other features discussed for phone and desktop: $60 \%$ Dist $_{B}$ values below 0.3 and $90 \%$ below 0.4 , which is not as good as $5(\mathrm{a})$ to $5(\mathrm{e})$. Feature is not considered for evaluation.

Figure 5(j) shows the CDF for AvgKeyHold has slightly better values for desktop, as about $60 \%$ of the samples have less than 0.4 Dist $_{B}$ and about $80 \%$ have less than 0.55 . There seems to be marginal improvement compared to other proposed features. On phone and tablet, this feature is slightly better than desktop, but not better than the features we already discussed for phone and 
Table 19. The Inter-User Dist $_{B}$ Values for StdFlight4 Distributions across All Devices

\begin{tabular}{|c|c|c|c|c|c|c|c|c|c|c|}
\hline Device & \multicolumn{3}{|c|}{ Desktop } & \multicolumn{3}{c|}{ Tablet } & \multicolumn{3}{c|}{ Phone } \\
\hline \hline Word & Mean & StD & Median & Mean & StD & Median & Mean & StD & Median \\
\hline are & 0.594 & 0.13 & 0.623 & 0.285 & 0.131 & 0.269 & 0.218 & 0.113 & 0.196 \\
\hline carefully & 0.025 & 0.038 & 0 & \multicolumn{3}{|c|}{-} & & 0.205 & 0.04 & 0.231 \\
\hline data & 0.202 & 0.099 & 0.202 & 0.05 & 0.086 & 0 & 0.139 & 0.132 & 0.101 \\
\hline different & 0.129 & 0.046 & 0.143 & 0.208 & 0.149 & 0.181 & \multicolumn{3}{c|}{-} \\
\hline first & 0.179 & 0.095 & 0.151 & 0.087 & 0.074 & 0.077 & \multicolumn{3}{c|}{-} \\
\hline have & 0.223 & 0.15 & 0.191 & 0.145 & 0.078 & 0.143 & 0.136 & 0.105 & 0.148 \\
\hline lines & 0.111 & 0.086 & 0.103 & 0.115 & 0.104 & 0.098 & 0.153 & 0.128 & 0.111 \\
\hline not & 0.437 & 0.143 & 0.42 & 0.237 & 0.143 & 0.227 & 0.123 & 0.102 & 0.099 \\
\hline overlap & 0.033 & 0.05 & 0 & 0.091 & 0.09 & 0.068 & & - & \\
\hline phase & 0.066 & 0.071 & 0.069 & 0.103 & 0.053 & 0.069 & 0.146 & 0.113 & 0.215 \\
\hline see & 0.573 & 0.102 & 0.569 & 0.292 & 0.129 & 0.274 & 0.139 & 0.09 & 0.147 \\
\hline that & 0.364 & 0.137 & 0.316 & 0.15 & 0.122 & 0.182 & 0.131 & 0.072 & 0.14 \\
\hline the & 0.67 & 0.131 & 0.685 & 0.236 & 0.147 & 0.224 & 0.27 & 0.135 & 0.275 \\
\hline there & 0.135 & 0.044 & 0.137 & 0.182 & 0.045 & 0.171 & 0.223 & 0.147 & 0.206 \\
\hline this & 0.331 & 0.132 & 0.308 & 0.183 & 0.107 & 0.175 & 0.211 & 0.129 & 0.206 \\
\hline two & 0.354 & 0.146 & 0.319 & 0.249 & 0.078 & 0.252 & 0.131 & 0.083 & 0.136 \\
\hline type & 0.294 & 0.104 & 0.27 & 0.098 & 0.098 & 0.088 & \multicolumn{3}{|c|}{-} \\
\hline will & 0.208 & 0.127 & 0.192 & 0.066 & 0.123 & 0 & 0.202 & 0.125 & 0.214 \\
\hline with & 0.2 & 0.126 & 0.161 & 0.137 & 0.106 & 0.117 & 0.089 & 0.092 & 0.076 \\
\hline words & 0.096 & 0.061 & 0.073 & 0.117 & 0.029 & 0.098 & 0.085 & 0.075 & 0.072 \\
\hline
\end{tabular}

desktop. About $60 \%$ of the Dist $_{B}$ values are below 0.3 and $90 \%$ are below 0.5 , which is not as good as 5(a) to 5(e). Feature is not considered for evaluation. Figure 5(k) shows the CDF for StdKeyHold, and we observe that it is the least discriminative among proposed features. For desktop, about $60 \%$ of the Dist $_{B}$ values are less than 0.6 and cover only $80 \%$ of the samples at a high Dist $_{B}$ value of 0.7 . This clearly implies that it is not very discriminative. Even in case of phone and tablet, $60 \%$ of samples have values below 0.6 , and $90 \%$ have Dist $_{B}$ values below 0.7 , which is not discriminative. This feature is not considered for evaluation.

\section{INFERENCE-PROPOSED FEATURES}

We find that these features separate user keystroke data better than the conventional features. We also eliminate 6 of the proposed 11 features. Among the 11 proposed features: WordHold, AvgFlight1, AvgFlight2, AvgFlight3, AvgFlight4, StdFlight1, StdFlight2, StdFlight3, StdFlight4, AvgKeyHold, and StdKeyHold. We find the most discriminative features to be: WordHold, AvgFlight1, AvgFlight2, AvgFlight3, and AvgFlight4. We use this subset of features for further evaluation, to build classifiers, and see if these features can provide competitive accuracies for user identification, which is discussed in the next section.

\section{EVALUATION}

From the analysis on the user separability of both classes of features (Conventional and Proposed), it is clear that the proposed features offered higher separability. We further selected a subset of the proposed features that clearly had higher discriminative power, such as WordHold, AvgFlight1, AvgFlight2, AvgFlight3, and AvgFlight4, and discarded the rest of our proposed features. We tested 
Table 20. The Inter-User Dist ${ }_{B}$ Values for StdKeyHold Distributions Across all Devices

\begin{tabular}{|c|c|c|c|c|c|c|c|c|c|c|}
\hline Device & \multicolumn{3}{|c|}{ Desktop } & \multicolumn{3}{c|}{ Tablet } & \multicolumn{3}{c|}{ Phone } \\
\hline \hline Word & Mean & StD & Median & Mean & StD & Median & Mean & StD & Median \\
\hline are & 0.497 & 0.183 & 0.495 & 0.507 & 0.152 & 0.508 & 0.454 & 0.165 & 0.479 \\
\hline carefully & 0.225 & 0.062 & 0.244 & \multicolumn{3}{|c|}{-} & & 0.245 & 0.283 & 0.133 \\
\hline data & 0.289 & 0.143 & 0.283 & 0.501 & 0.133 & 0.524 & 0.398 & 0.156 & 0.397 \\
\hline different & 0.291 & 0.267 & 0.172 & 0.355 & 0.185 & 0.367 & \multicolumn{3}{c|}{-} \\
\hline first & 0.455 & 0.086 & 0.428 & 0.517 & 0.121 & 0.499 & \multicolumn{3}{c|}{-} \\
\hline have & 0.277 & 0.121 & 0.286 & 0.31 & 0.183 & 0.319 & 0.394 & 0.223 & 0.452 \\
\hline lines & 0.19 & 0.131 & 0.212 & 0.413 & 0.116 & 0.416 & 0.265 & 0.185 & 0.3 \\
\hline not & 0.344 & 0.235 & 0.405 & 0.501 & 0.165 & 0.529 & 0.381 & 0.109 & 0.399 \\
\hline overlap & 0.371 & 0.141 & 0.345 & 0.331 & 0.255 & 0.273 & & - & \\
\hline phase & 0.312 & 0.206 & 0.369 & 0.266 & 0.154 & 0.338 & 0.477 & 0.086 & 0.456 \\
\hline see & 0.564 & 0.17 & 0.596 & 0.376 & 0.134 & 0.347 & 0.478 & 0.103 & 0.465 \\
\hline that & 0.427 & 0.138 & 0.448 & 0.308 & 0.126 & 0.378 & 0.494 & 0.116 & 0.497 \\
\hline the & 0.635 & 0.124 & 0.634 & 0.477 & 0.147 & 0.485 & 0.412 & 0.135 & 0.425 \\
\hline there & 0.283 & 0.103 & 0.271 & 0.193 & 0.081 & 0.237 & 0.509 & 0.094 & 0.521 \\
\hline this & 0.426 & 0.122 & 0.436 & 0.509 & 0.125 & 0.527 & 0.464 & 0.186 & 0.494 \\
\hline two & 0.632 & 0.166 & 0.644 & 0.46 & 0.155 & 0.481 & 0.367 & 0.068 & 0.372 \\
\hline type & 0.262 & 0.124 & 0.241 & 0.356 & 0.16 & 0.346 & & - & \\
\hline will & 0.222 & 0.215 & 0.085 & 0.378 & 0.147 & 0.324 & 0.323 & 0.179 & 0.335 \\
\hline with & 0.335 & 0.176 & 0.351 & 0.394 & 0.137 & 0.387 & 0.307 & 0.234 & 0.329 \\
\hline words & 0.255 & 0.095 & 0.26 & 0.371 & 0.061 & 0.392 & 0.435 & 0.119 & 0.445 \\
\hline
\end{tabular}

these features by building classifiers based on them. We are using similar methodology as used by Janakiraman and Sim [25], Sim and Janakiraman [48].

To evaluate our proposed features and to measure the improvement in user identification achieved with them, we build classifiers with both conventional features and proposed features. We then compare their results for the task of user identification. The underlying principle of the classifier is same for both the cases. The known histograms $H_{k}$ of each person are compared to the histograms built from the input text $H_{i}$. We again use Bhattacharyya distance as described before, but this time, we are looking for maximum overlap (max value between 0 to 1 ) between the two histograms. We take the average histogram overlap from all the features and identify the person as the one with the highest overlap. For conventional features, we built classifiers for each of the Di-graphs discussed earlier. For a Di-graph with characters $d_{i}$ and $d_{i+1}$, we use the argument maximize classifier represented by Equation (2) as our classifier to identify the user.

Argument maximize classifier using conventional features for a Di-graph $d_{i} d_{i+1}$ :

$$
\begin{array}{r}
\underset{u}{\operatorname{argmax}} \operatorname{Avg}\left(\text { Dist }_{B}\left(H_{k d_{i} \text { KeyHold }}, H_{i} d_{i} \text { KeyHold }\right),\right. \\
\text { Dist }_{B}\left(H_{k d_{i+1} \text { KeyHold }}, H_{i} d_{i+1} \text { KeyHold }\right), \\
\operatorname{Dist}_{B}\left(H_{k d_{i} d_{i+1} \text { Flight } 1}, H_{i} d_{i} d_{i+1} \text { Flight } 1\right), \\
\text { Dist }_{B}\left(H_{k d_{i} d_{i+1} \text { Flight } 2}, H_{i} d_{i} d_{i+1} \text { Flight } 2\right), \\
\text { Dist }_{B}\left(H_{k d_{i} d_{i+1} \text { Flight } 3}, H_{i} d_{i} d_{i+1} \text { Flight } 3\right), \\
\left.\operatorname{Dist}_{B}\left(H_{k d_{i} d_{i+1} \text { Flight } 4}, H_{i} d_{i} d_{i+1} \text { Flight } 4\right)\right) .
\end{array}
$$




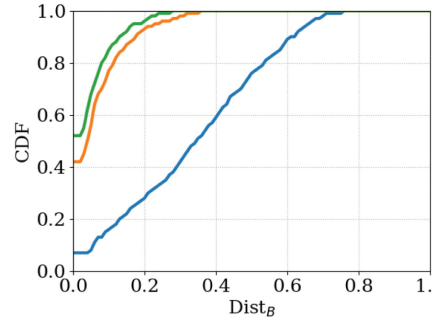

(a) WordHold

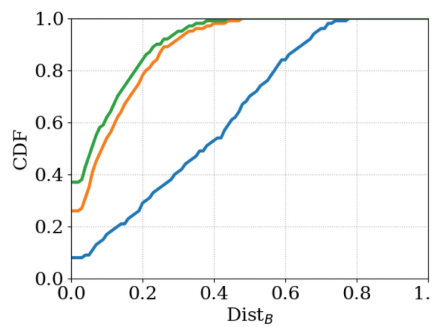

(d) AvgFlight3

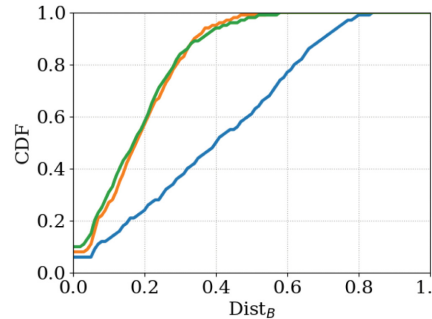

(g) StdFlight2

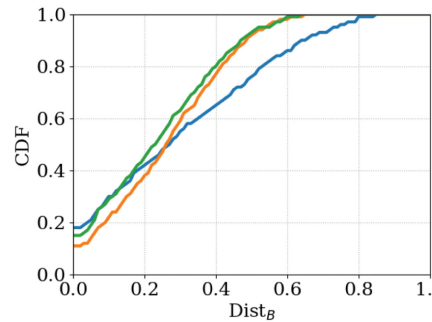

(j) AvgKeyHold

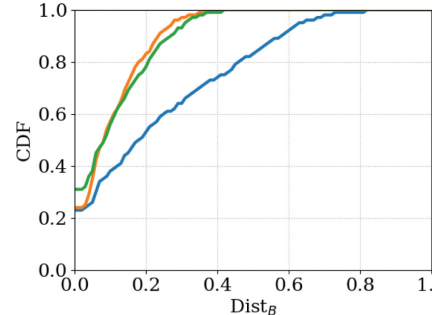

(b) AvgFlight1

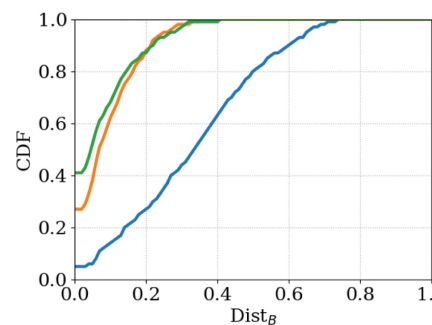

(e) AvgFlight4

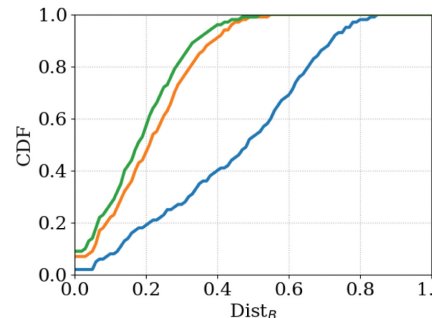

(h) StdFlight3

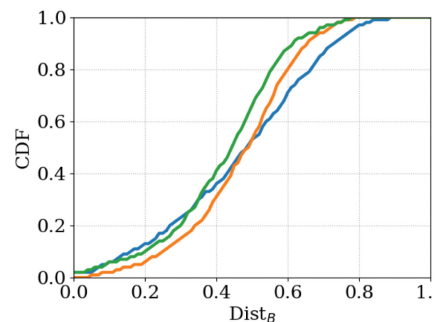

(k) StdKeyHold

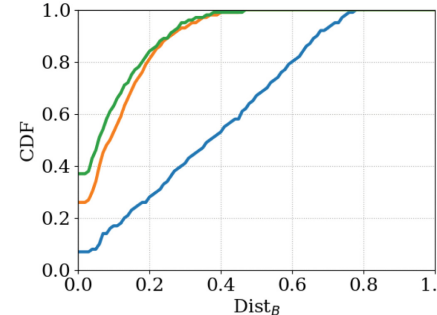

(c) AvgFlight2

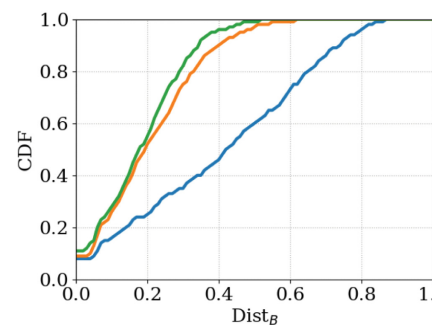

(f) StdFlight1

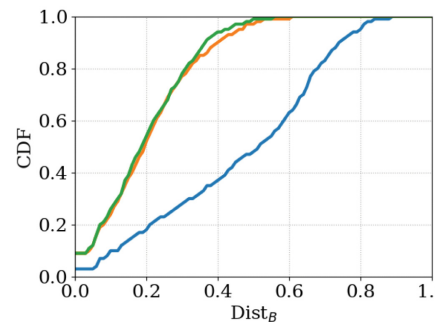

(i) StdFlight4

Fig. 5. Comparing the Bhattacharyya distances of PDFs for all proposed context-sensitive features on desktop, tablet, and phone.

Argument maximize classifier using proposed features for a word $X$ :

$$
\begin{aligned}
& \operatorname{argmax} A v g\left(\text { Dist }_{B}\left(H_{k} \text { XWordHold }, H_{i} \text { XWordHold }\right)\right. \text {, } \\
& { }^{u} \operatorname{Dist}_{B}\left(H_{k X A v g-F l i g h t 1}, H_{i} \text { XavgFlight } 1\right) \text {, } \\
& \operatorname{Dist}_{B}\left(H_{k X \text { Avg-Flight } 2}, H_{i X A v g-F l i g h t 2}\right) \text {, } \\
& \operatorname{Dist}_{B}\left(H_{k} \text { XAvg-Flight } 3, H_{i} \text { XAvg-Flight } 3\right) \text {, } \\
& \left.\operatorname{Dist}_{B}\left(H_{k X A v g-F l i g h t}, H_{i} \text { XAvg-Flight } 4\right)\right) \text {. }
\end{aligned}
$$


Table 21. Classifier Accuracies for the Conventional Feature-based Classifiers in Our Experiment

\begin{tabular}{|c|c|c|c|c|c|c|}
\hline Device & \multicolumn{2}{|c|}{ Desktop } & \multicolumn{2}{|c|}{ Tablet } & \multicolumn{2}{|c|}{ Phone } \\
\hline Digraph & Mean & Std. dev. & Mean & Std. dev. & Mean & Std. dev. \\
\hline (“space," “a”) & 0.63 & 0.04 & 0.76 & 0.12 & 0.8 & 0.097 \\
\hline ("space," "i") & 0.68 & 0.04 & 0.81 & 0.163 & 0.83 & 0.08 \\
\hline ("space," "s") & 0.58 & 0.053 & 0.79 & 0.091 & 0.8 & 0.105 \\
\hline ("space," “t”) & 0.42 & 0.07 & 0.69 & 0.053 & 0.76 & 0.093 \\
\hline (“e," "space") & 0.44 & 0.032 & 0.59 & 0.06 & 0.67 & 0.075 \\
\hline ("e," "r") & 0.55 & 0.035 & 0.66 & 0.055 & 0.7 & 0.064 \\
\hline ("e," "s") & 0.52 & 0.071 & 0.62 & 0.087 & 0.68 & 0.077 \\
\hline (“o," “n”) & 0.69 & 0.045 & 0.67 & 0.044 & 0.72 & 0.069 \\
\hline ("r," "e") & 0.53 & 0.06 & 0.58 & 0.053 & 0.59 & 0.062 \\
\hline ("t," "space") & 0.56 & 0.063 & 0.62 & 0.08 & 0.71 & 0.092 \\
\hline ("t," "h") & 0.49 & 0.03 & 0.58 & 0.035 & 0.66 & 0.056 \\
\hline
\end{tabular}

For the proposed features, we used an argument maximize classifier represented by Equation (3) as our classifier for a word $X$. Using the most common unigraphs and Di-graphs discussed in the previous sections, we built 11 classifiers that used the conventional features and 20 classifiers, one for each word considered in the previous section, which used our proposed features. We used a Synthetic Minority Over-sampling Technique (SMOTE), as described in Chawla et al. [11], to balance and oversample our data when needed, and use 10-fold cross validation and report the mean and standard deviation of the accuracies of our classifiers. As our data were balanced and we had 20 users, the chance of random guess identification was 0.05 . Tables 21 and 22 present the accuracies of all the classifiers. In case of the conventional features, we can observe low accuracies in most cases. Except for a few Di-graphs in case of hand-held devices ((space,i), (space,a), (space,s)), accuracies range from $45 \%$ to $75 \%$, which is not very desirable. The accuracy of classifiers is extremely low in case of the desktop with conventional features, with the highest accuracy being $68 \%$ for the Di-graph (space,i). Whereas, while using the classifiers with proposed subset of features, we see that most accuracies lie in the range of $87 \%$ to $97 \%$. With desktop, we found that classifiers for the words: "data," "first," "have," and "that" had accuracies of over $90 \%$, while many others performed fairly well with accuracies above $85 \%$. Even the worst performer, "type," was $73.2 \%$ accurate. Both hand-held devices have high accuracies for a majority of the words selected. On tablet, the classifiers built for words: "see," "that," "there," and "with" had accuracies of 95\% and above; lowest accuracies was for the word "two," at 75.3\%. On phone, the classifiers built for words: "data," "have," "see," "this," and "with" had accuracies of above 93\%. The lowest accuracy on phone was for the classifier of the word "phase," at $85 \%$.

\section{INSIGHTS}

We show that the conventional features have lower discriminative power between users when compared to the proposed context-sensitive features. This leads to many more important and intriguing questions:

\subsection{Insight 1: Why do the Proposed Features Perform Better than the Conventional Features?}

To answer this question, we use the analysis of Entropy as a measure of disorder in data and explain why proposed features tend to perform better than the conventional features. To be more 
Table 22. Classifier Accuracies for the Proposed Feature-based Classifiers in Our Experiment

\begin{tabular}{|c|l|c|c|c|c|c|}
\hline Device & \multicolumn{2}{|c|}{ Desktop } & \multicolumn{2}{c|}{ Tablet } & \multicolumn{2}{c|}{ Phone } \\
\hline \hline Word & Mean & Std. dev. & Mean & Std. dev. & Mean & Std. dev. \\
\hline are & 0.85 & 0.071 & 0.87 & 0.056 & 0.91 & 0.054 \\
\hline carefully & 0.86 & 0.063 & - & - & 0.89 & 0.051 \\
\hline data & 0.93 & 0.056 & 0.915 & 0.055 & 0.975 & 0.034 \\
\hline different & 0.88 & 0.046 & 0.875 & 0.06 & - & - \\
\hline first & 0.93 & 0.033 & 0.84 & 0.073 & - & - \\
\hline have & 0.905 & 0.035 & 0.91 & 0.07 & 0.963 & 0.034 \\
\hline lines & 0.875 & 0.046 & 0.85 & 0.036 & 0.91 & 0.044 \\
\hline not & 0.895 & 0.057 & 0.91 & 0.049 & 0.915 & 0.05 \\
\hline overlap & 0.875 & 0.06 & 0.868 & 0.049 & - & - \\
\hline phase & 0.875 & 0.068 & 0.84 & 0.03 & 0.859 & 0.06 \\
\hline see & 0.895 & 0.035 & 0.96 & 0.037 & 0.935 & 0.039 \\
\hline that & 0.905 & 0.072 & 0.95 & 0.039 & 0.911 & 0.053 \\
\hline the & 0.785 & 0.045 & 0.865 & 0.055 & 0.932 & 0.041 \\
\hline there & 0.895 & 0.052 & 0.963 & 0.034 & 0.911 & 0.075 \\
\hline this & 0.889 & 0.05 & 0.875 & 0.068 & 0.945 & 0.042 \\
\hline two & 0.86 & 0.037 & 0.753 & 0.058 & 0.865 & 0.059 \\
\hline type & 0.732 & 0.055 & 0.821 & 0.042 & - & - \\
\hline will & 0.885 & 0.071 & 0.87 & 0.046 & 0.889 & 0.064 \\
\hline with & 0.89 & 0.07 & 0.95 & 0.039 & 0.935 & 0.055 \\
\hline words & 0.868 & 0.063 & 0.86 & 0.062 & 0.863 & 0.059 \\
\hline
\end{tabular}

precise, the information gain-which is a measure of the decrease in disorder that is achieved by partitioning the data-is the key concept being exploited with the help of our proposed features. Let $x$ be a unigraph or a Di-graph. In the conventional approach, features values associated with $x$ (Keyhold if $\mathrm{x}$ is a unigraph, Flight $t_{1}$ through Flight 4 if $\mathrm{x}$ is a Di-graph) are grouped together irrespective of context. Let $\vec{d}_{x}$ represent vector of feature values extracted for $x$ from text sample. The entropy of $\vec{d}_{x}$ can be expressed as:

$$
E\left(\vec{d}_{x}\right)=-\sum_{i=1}^{k} p_{i} \log _{2}\left(p_{i}\right),
$$

where $i=1$ to $k$ are the number of bins into which $\vec{d}_{x}$ is split and $p_{i}$ is the probability of the feature value of $x$ being in bin $i$. Practically, the values forming $\vec{d}_{x}$ come from different contexts or words, as described in previous sections. Let $\vec{w}$ represent the vector of different words from which the values of $\vec{d}_{x}$ are extracted. Therefore, if $\vec{d}_{x}$ were to be partitioned based on the context from which its values come from, the entropy of such partition can be expressed as:

$$
E\left(\vec{d}_{x}, \vec{w}\right)=\sum_{j=1}^{m}\left(\frac{n_{j}}{n} x E\left(\vec{d}_{x_{j}}\right)\right),
$$

where $j=1$ to $m$ are the number of different words that values of $x$ are extracted from. Essentially, Equation (5) gives us the sum of the weighted average of the entropy from all the partitions. The information gained by performing this partition-or in other words, the reduction in disorder 
Table 23. Example from Our Desktop Dataset: Average Feature Values for a Randomly Chosen User Shows the Variations in the Average Feature Values for the Character "h" and Digraph "ha"

Depending on the Context in which they Appear

\begin{tabular}{|c|c|c|}
\hline & $\begin{array}{c}\text { Avg. KeyHold } \\
\text { time of "h" }\end{array}$ & $\begin{array}{c}\text { Avg. [Flight1, Flight2, Flight3, Flight4] } \\
\text { time of "ha" }\end{array}$ \\
\hline \hline Over the entire sample & 183.45 & {$[46.6,270.2,241.2,470.0]$} \\
\hline Over all occurrences of "that" & 167.20 & {$[12.4,230.0,201.3,389.5]$} \\
\hline Over all occurrences of "have" & 189.15 & {$[44.7,279.6,252.0,461.8]$} \\
\hline
\end{tabular}

All values are in milliseconds.

achieved by the partitioning-can be expressed as:

$$
I\left(\vec{d}_{x}, \vec{w}\right)=E\left(\vec{d}_{x}\right)-E\left(\vec{d}_{x}, \vec{w}\right) .
$$

Therefore, theoretically, $I\left(\vec{d}_{x}, \vec{w}\right) \geq 0$, but, practically, the cases where $I\left(\vec{d}_{x}, \vec{w}\right)=0$ can only occur if $\vec{w}$ partitions $\vec{d}_{x}$ into partitions with the same probabilities as $\vec{d}_{x}$. Since $\vec{w}$ inherently consists of only high-frequency words, the chances of $E\left(\vec{d}_{x}\right)=E\left(\vec{d}_{x}, \vec{w}\right)$ are not practical and hence $I\left(\vec{d}_{x}, \vec{w}\right)=0$ is highly unlikely. Therefore, however small the difference between $E\left(\vec{d}_{x}\right)$ and $E\left(\vec{d}_{x}, \vec{w}\right)$ may be, it is highly likely to lead to a positive information gain, or reduction of disorder in data, which in our case are the feature values. Reduction in disorder means the partitions have much more homogeneous values than when taken without the partition, which intuitively should be better for classification or identification. We observed that entropy of the feature values when extracted with context restrictions were much lower than a global extraction approach. Because $I\left(\vec{d}_{x}, \vec{w}\right) \geq 0$, and the case of $I\left(\vec{d}_{x}, \vec{w}\right)=0$ is impractical, this answers our question about why the proposed features work better than the conventional features.

An example of how the feature values vary with respect to context is shown in Table 23, where we chose a random user from our dataset and calculated the average feature values for character "h" and Di-graph "ha," first, over all occurrences, then, only over all occurrences in the words "that" and "have" separately. When overall occurrences are computed, the average key-hold time for " $h$ " was about $183.5 \mathrm{~ms}$, which is much higher than key-hold time $167.2 \mathrm{~ms}$, in all occurrences of "that." The average Flight1 values of "ha" for all occurrences of "that" and "have" were $12.4 \mathrm{~ms}$ and $44.7 \mathrm{~ms}$, respectively. Similar distinction can be found in all feature values; as shown in Table 23, it is representative of a majority of the dataset for all users. From this example, it is clear that context affects the feature values.

\subsection{Insight 2: Why are the Results and the Performance of Proposed Features Better in Case of Hand-held Devices When Compared to the Desktop?}

To help answer this question, we look for inherent differences between the devices, their usage, and the data collected on them. There are several reasons that we suspect might lead to the difference in the efficiency of the features. First, the very nature of the usage of these devices is different. As shown in Figure 6(a), a typical keyboard when used on a table allows only two degrees of freedom, forward/backward and left/right. In comparison, a mobile phone offers six degrees of freedom; forward/backward, left/right, upward/downward, yaw, pitch, and roll, as shown in Figure 6(b). This can affect the style of using a keyboard and hence bring in some differences in data collected from these families of devices.

Another reason for this difference in effectiveness of features, we suspect, is the consistency in the typing speeds. We found that a majority of the users had larger standard deviations in the time required to type a complete word on the desktop when compared to the hand-held devices. 


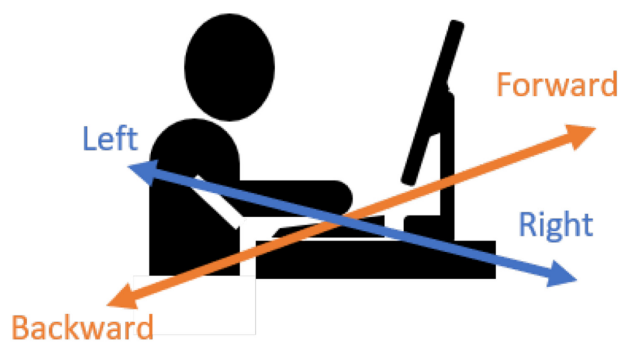

(a) Degrees of freedom while typing on a desktop.

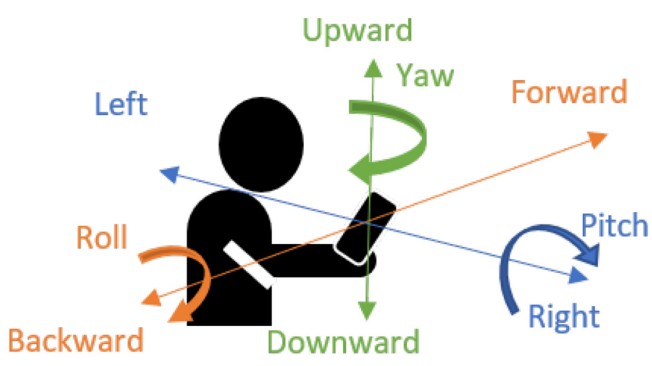

(b) Degrees of freedom while typing on a phone.

Fig. 6. Typically, a desktop keyboard offers only two degrees of freedom; forward/backward and left/right. As a typical phone can be held by its user in any comfortable posture, it offers six degrees of freedom; forward/backward, left/right, upward/downward, yaw, pitch, and roll, as shown in these figures.

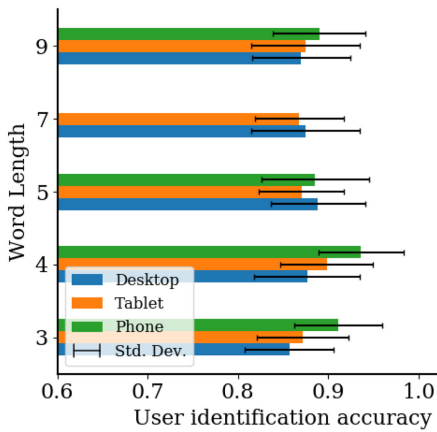

(a)

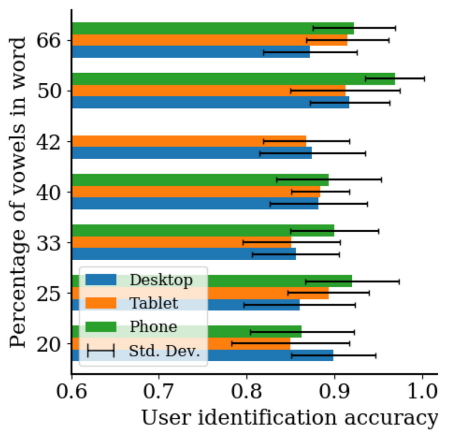

(b)

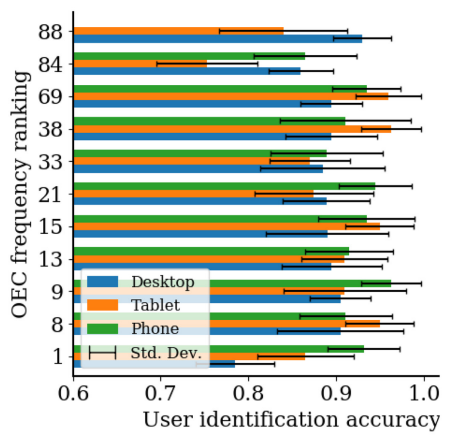

(c)

Fig. 7. Impact of three word-based factors on the performance of proposed features for user identification. The three factors are: Word length (Figure 7(a)): number of characters in a word; Vowel Percentage (Figure 7(b)): percentage of vowels in a word; and Oxford English Corpus (OEC) frequency ranking (Figure $7(\mathrm{c})$ ): the frequency ranking of the words in our study according to OEC (Top 100). The words in order of rank (Figure $7(\mathrm{c}), \mathrm{y}$-axis) are: (1, the), (8, that), (9, have), (13, not), (15, with), (21, this), (33, will), (38, there), $(69$, see), $(84$, two $)$, and (88, first).

In particular, our proposed feature "WordHold" reflected this behavior for most of the users. The standard deviation for WordHold of a word on the hand-held devices was about three-quarters that of the standard deviation of WordHold of the same word on desktop typed by the same user. We also observed that the KeyHold duration for "space" between words and the Flight 1 duration from "space" to the first letter of words were more densely clustered in case of the desktop when compared to the hand-held devices. On hand-held devices, this behavior gave the impression of small uniform bursts of typing activity (word) followed by nonuniform pauses (space). In contrast, typing behavior on desktops appeared to be nonuniform throughout. As all users in our study owned smart-phones and indicated that their usage of phones was much higher than their usage of desktops, we posit that it leads to these typing patterns on different devices.

\subsection{Insight 3: What Word-based Factors Might Impact the User Identification Performance of Proposed Features?}

Figure 7 shows the impact of three word-based factors: (1) word length; (2) percentage of vowels in the word; and (3) Oxford English Corpus (OEC) frequency ranking (top 100) [41] on the 
performance of the proposed features for user identification (Table 22). Short words (three to four characters) performed slightly better on hand-held devices, whereas medium-length words (five to seven characters) performed slightly better on the desktop, as shown in Figure 7(a). For the percentage of vowels in a word, we observe a gradual improvement in accuracy until $50 \%$ of the word is composed of vowels (Figure 7(b)). For all three types of devices, the identification accuracy peaks when the words to extract features have $50 \%$ vowels. The ranking (Figure $7(\mathrm{c})$ ) consists of words in our study that are also most frequent (top 100) in the Oxford English Corpus (OEC) [41] and the Corpus of Contemporary American English (COCA) [15]. The medium-length words (four to five characters), words with about $50 \%$ vowel composition, and those that are ranked higher in frequency give the best results for user identification using the proposed features. The word "the" is an exception to these observations, because it performs poorly on the desktop and tablet even though it is the most-used word in the corpora.

\subsection{Discussion: Attacks and Limitations}

The most common types of attacks on text in the literature are: (1) for inferring typed text (generally PIN and Password through side-channel attacks) or (2) for inferring keystroke timings (generally impersonation attacks). We discuss both in the following paragraphs.

Inferring typed text. Side-channel attacks, such as video [12, 47], smartwatch [32], acoustic signals [31], vibrations in video [26], and Channel State Information [2, 18] use some form of eavesdropping to obtain the victim's typed text rather than impersonating their typing behavior. The domain of these attacks does not address keystroke timings, so they are not applicable to our scenarios.

Inferring keystroke timings. Attacks to mimic the keystroke timings of a victim are forms of presentation attacks, where typically the attacker formulates an imposter text sample (including the keystroke timings) from data drawn from the statistics of acquired samples [42, 45], either stolen or from publicly available databases. Typically, these attacks use conventional features and assume the latencies to be similar across the entire keystroke data; however, our analysis and examples (Table 23) show that keystroke latencies vary, depending on the context that the keys appear in. Though similar methods could be applied to attack our proposed features, the obstacles to factor in the context of keys would be significant. Additionally, Stefan and Yao [49] and Huang et al. [23] also provide measures to defend against such attacks. Another attack presented by Khan et al. [28] explored Augmented Reality (AR) to mimic a user's typing with assistance from the AR system. Apart from using conventional features, the attack made several non-trivial assumptions regarding the availability of the victim's device and keystroke timings. The settings of this attack are complex and difficult in real-life scenarios; however, applicability, although unlikely to our proposed features, needs exploration. The viability of an attack when an attacker obtains both the typed text and the keystroke timings is an open problem that needs exploration.

\subsection{Conclusion and Future Work}

We show that proposed word-specific features perform much better at user identification on all devices. Conventional features, especially KeyHold, does not provide user separation to a desired level. We considered the subset of proposed features that offered higher discriminability, such as WordHold, AvgFlight1, AvgFlight2, AvgFlight3, and AvgFlight4, evaluated them with classifiers, and drew comparisons with conventional features (Section 9). These classifiers show competitive accuracies on all devices. Mathematical insights for this improvement in performance are drawn (Section 10.1). We also note that these features in general perform much better on hand-held devices. We speculate that users' style of holding devices and patterns, such as short bursts of typing followed by pauses between words, might be some of the reasons (Section 10.2). Analysis of the 
word-based impact factors reveal that four- or five-character words, words with about $50 \%$ vowels, and those that are ranked higher on the frequency lists might give better results for the extraction and use of the proposed features (Section 10.3) for user identification.

The results of our experiment call for a shift from conventional features to word-specific features for continuous authentication using $\mathrm{KD}$. We are of the opinion that factoring in the knowledge of context can be beneficial to $\mathrm{KD}$. We hope this article provides direction to researchers of optimum $\mathrm{KD}$ features. As part of our future work, we are exploring inter-device relationships in KD to see how a user's behavior on a device is linked to their behavior on another.

\section{APPENDICES}

A COGNITIVE LOADS [10]

\begin{tabular}{|c|c|c|}
\hline Task & Level & Required activity \\
\hline Remember & 1 & Retrieve knowledge from long-term memory to explain \\
\hline Understand & 2 & Explain, summarize, or interpret \\
\hline Apply & 3 & Apply, execute, or implement \\
\hline Analyze & 4 & Organize or break material into constituent parts \\
\hline Evaluate & 5 & Critique or make judgments based on criteria \\
\hline Create & 6 & Generate, plan, or put elements together \\
\hline
\end{tabular}

\section{B EXAMPLES OF FREE-TEXT QUESTIONS ON DESKTOP}

- List some of the things that you like about Syracuse University.

- Which internet browser do you typically use (e.g, Google Chrome, Internet Explorer, Mozilla Firefox)?

- Give step-by-step driving directions to your favorite restaurant in the Syracuse Area, starting from your dorm room/ home.

- Discuss step-by-step instructions for making your favorite type of sandwich. Write them so the person who has never done this before can follow your instructions.

\section{EXAMPLES OF FREE-TEXT QUESTIONS ON TABLET}

- What is your ideal job after graduation? Why?

- Why did you decide to attend Syracuse University?

- Review Question \#6 (from the Multiple-Choice Questions section) and the answer that you chose. Why did you select your answer?

- If Question \#6 (from the Multiple-Choice Questions section) was changed to read "If some mangoes are golden in color and no golden-colored things are cheap," which answer would be correct and why?

\section{EXAMPLES OF FREE-TEXT QUESTIONS ON PHONE}

- Of the courses you've taken in college, which was your favorite and why?

- Think about a class that you did not enjoy. What improvements would you like to see to make the course better?

- Do you intend to pursue an advanced degree (e.g., Master's or Ph.D.)? Why or why not?

- Review Question \#7 (from the Multiple-Choice Questions section) and the answer that you chose. Why was the rule you found/why did you select your answer? 


\section{E TRANSCRIPTION SENTENCES}

- "this is a test to see if the words that $\mathrm{i}$ type are unique to me. there are two sentences in this data sample." ${ }^{1}$

- "second session will have different set of lines. carefully selected not to overlap with the first collection phase."1

\section{REFERENCES}

[1] Ahmed A. Ahmed and Issa Traore. 2014. Biometric recognition based on free-text keystroke dynamics. IEEE Trans. Cyber. 44, 4 (2014).

[2] Kamran Ali, Alex X. Liu, Wei Wang, and Muhammad Shahzad. 2015. Keystroke recognition using Wi-Fi signals. In Proceedings of the 21st International Conference on Mobile Computing and Networking (MobiCom'15). ACM, New York, NY, 90-102. DOI : https://doi.org/10.1145/2789168.2790109

[3] Md Liakat Ali, John V. Monaco, Charles C. Tappert, and Meikang Qiu. 2017. Keystroke biometric systems for user authentication. F. Sig. Proc. Syst. 86, 2 (1 Mar. 2017), 175-190. DOI : https://doi.org/10.1007/s11265-016-1114-9

[4] Orcan Alpar. 2017. Frequency spectrograms for biometric keystroke authentication using neural network based classifier. Knowledge-Based Systems 116 (2017), 163-171. DOI : https://doi.org/10.1016/j.knosys.2016.11.006

[5] Arwa Alsultan, Kevin Warwick, and Hong Wei. 2017. Non-conventional keystroke dynamics for user authentication. Pattern Recog. Lett. 89, C (2017).

[6] Dmitri Asonov and Rakesh Agrawal. 2004. Keyboard acoustic emanations. In Proceedings of the IEEE Symposium on Security and Privacy (S\&P'04). 3-11. DOI : https://doi.org/10.1109/SECPRI.2004.1301311

[7] Gabriel L. F. B. G. Azevedo, George D. C. Cavalcanti, and Edson C. B. Carvalho Filho. 2007. An approach to feature selection for keystroke dynamics systems based on PSO and feature weighting. In Proceedings of the IEEE Congress on Evolutionary Computation (CEC'07). IEEE, 3577-3584.

[8] Kiran Balagani, Vir Phoha, Asok Ray, and Shashi Phoha. 2011. On the discriminability of keystroke feature vectors used in fixed text keystroke authentication. Pattern Recog. Lett. 32, 7 (2011).

[9] Jorge Blasco, Thomas M. Chen, Juan Tapiador, and Pedro Peris-Lopez. 2016. A survey of wearable biometric recognition systems. ACM Comput. Surv. 49, 3, Article 43 (Sept. 2016), 35 pages. DOI : https://doi.org/10.1145/2968215

[10] David Guy Brizan, Adam Goodkind, Patrick Koch, Kiran Balagani, Vir V. Phoha, and Andrew Rosenberg. 2015. Utilizing linguistically enhanced keystroke dynamics to predict typist cognition and demographics. Int. f. Hum.-Comput. Stud. 82 (2015), 57-68. DOI : https://doi.org/10.1016/j.ijhcs.2015.04.005

[11] Nitesh V. Chawla, Kevin W. Bowyer, Lawrence O. Hall, and W. Philip Kegelmeyer. 2002. SMOTE: Synthetic minority over-sampling technique. J. Artif. Int. Res. 16, 1 (June 2002), 321-357. Retrieved from: http://dl.acm.org/citation.cfm? id $=1622407.1622416$.

[12] Y. Chen, T. Li, R. Zhang, Y. Zhang, and T. Hedgpeth. 2018. EyeTell: Video-assisted touchscreen keystroke inference from eye movements. In Proceedings of the IEEE Symposium on Security and Privacy (SP'18). 144-160. DOI : https:// doi.org/10.1109/SP.2018.00010

[13] F. Ciuffo and G. M. Weiss. 2017. Smartwatch-based transcription biometrics. In Proceedings of the IEEE 8th Ubiquitous Computing, Electronics and Mobile Communication Conference (UEMCON'17). 145-149. DOI : https://doi.org/10.1109/ UEMCON.2017.8249014

[14] N. L. Clarke and S. M. Furnell. 2006. Authenticating mobile phone users using keystroke analysis. Int. f. Inf. Secur. 6, 1 (2006).

[15] Mark Davies. 2010. Corpus of contemporary American English. Retrieved from: https://www.english-corpora.org/ coca/.

[16] H. Davoudi and E. Kabir. 2009. A new distance measure for free text keystroke authentication. In Proceedings of the 14th International CSI Computer Conference. 570-575. DOI : https://doi.org/10.1109/CSICC.2009.5349640

[17] K. Delac and M. Grgic. 2004. A survey of biometric recognition methods. In Proceedings of the 46th International Symposium on Electronics in Marine (Elmar'04). 184-193.

[18] Song Fang, Ian Markwood, Yao Liu, Shangqing Zhao, Zhuo Lu, and Haojin Zhu. 2018. No training hurdles: Fast training-agnostic attacks to infer your typing. In Proceedings of the ACM SIGSAC Conference on Computer and Communications Security. ACM, 1747-1760.

\footnotetext{
${ }^{1}$ The transcription sentences were selected based on two criteria: (1) inclusion of many frequently used words in the Oxford English Corpus, and (2) encouraging typing activity on both hands (on both sides on the keyboard). Transcription sentences were typed in lower case.
} 
[19] Adam Goodkind, David Guy Brizan, and Andrew Rosenberg. 2017. Utilizing overt and latent linguistic structure to improve keystroke-based authentication. Image Vis. Comput. 58 (2017), 230-238. DOI : https://doi.org/10.1016/j.imavis. 2016.06.003

[20] Daniele Gunetti and Claudia Picardi. 2005. Keystroke analysis of free text. ACM Trans. Inf. Syst. Secur. 8, 3 (Aug. 2005), 312-347. DOI : https://doi.org/10.1145/1085126.1085129

[21] Daniele Gunetti, Claudia Picardi, and Giancarlo Ruffo. 2005. Dealing with different languages and old profiles in keystroke analysis of free text. In $A I^{*} I A$ 2005: Advances in Artificial Intelligence. Springer Berlin Heidelberg, 347-358.

[22] Jiacang Ho and Dae-Ki Kang. 2017. Mini-batch bagging and attribute ranking for accurate user authentication in keystroke dynamics. Pattern Recog. 70 (2017), 139-151. DOI : https://doi.org/10.1016/j.patcog.2017.05.002

[23] J. Huang, D. Hou, and S. Schuckers. 2017. A practical evaluation of free-text keystroke dynamics. In Proceedings of the IEEE International Conference on Identity, Security and Behavior Analysis (ISBA'17). 1-8. DOI : https://doi.org/10.1109/ ISBA.2017.7947695

[24] Jiaju Huang, Daqing Hou, Stephanie Schuckers, and Shambhu Upadhyaya. 2016. Effects of text filtering on authentication performance of keystroke biometrics. In Proceedings of the IEEE International Workshop on Information Forensics and Security (WIFS'16).

[25] Rajkumar Janakiraman and Terence Sim. 2007. Keystroke dynamics in a general setting. In Advances in Biometrics, Seong-Whan Lee and Stan Z. Li (Eds.). Springer Berlin, 584-593.

[26] K. Jin, S. Fang, C. Peng, Z. Teng, X. Mao, L. Zhang, and X. Li. 2017. ViViSnoop: Someone is snooping your typing without seeing it! In Proceedings of the IEEE Conference on Communications and Network Security (CNS'17). 1-9. DOI : https://doi.org/10.1109/CNS.2017.8228624

[27] Rick Joyce and Gopal Gupta. 1990. Identity authentication based on keystroke latencies. Commun. ACM 33, 2 (Feb. 1990), 168-176. DOI : https://doi.org/10.1145/75577.75582

[28] Hassan Khan, Urs Hengartner, and Daniel Vogel. 2018. Augmented reality-based mimicry attacks on behaviour-based smartphone authentication. In Proceedings of the 16th International Conference on Mobile Systems, Applications, and Services (MobiSys'18). ACM, New York, NY, 41-53. DOI : https://doi.org/10.1145/3210240.3210317

[29] H. S. Lee, T. S. Lau, W. K. Lai, Y. C. King, and L. L. Lim. 2017. User identification of numerical keypad typing patterns with subtractive clustering fuzzy inference. In Proceedings of the IEEE 15th Student Conference on Research and Development (SCOReD'17). 83-88. DOI : https://doi.org/10.1109/SCORED.2017.8305416

[30] Jian Liu, Yan Wang, Gorkem Kar, Yingying Chen, Jie Yang, and Marco Gruteser. 2015. Snooping Keystrokes with Mm-level audio ranging on a single phone. In Proceedings of the 21st International Conference on Mobile Computing and Networking (MobiCom'15). ACM, New York, NY, 142-154. DOI : https://doi.org/10.1145/2789168.2790122

[31] L. Lu, J. Yu, Y. Chen, Y. Zhu, X. Xu, G. Xue, and M. Li. 2019. KeyLiSterber: Inferring keystrokes on QWERTY keyboard of touch screen through acoustic signals. In Proceedings of the IEEE Conference on Computer Communications (INFOCOM'19). 775-783. DOI : https://doi.org/10.1109/INFOCOM.2019.8737591

[32] A. Maiti, M. Jadliwala, J. He, and I. Bilogrevic. 2018. Side-channel inference attacks on mobile keypads using smartwatches. IEEE Trans. Mob. Comput. 17, 9 (Sept. 2018), 2180-2194. DOI : https://doi.org/10.1109/TMC.2018. 2794984

[33] Merylin Monaro, Chiara Galante, Riccardo Spolaor, Qian Qian Li, Luciano Gamberini, Mauro Conti, and Giuseppe Sartori. 2018. Covert lie detection using keyboard dynamics. Sci. Rep. 8, 1 (2018), 1976. DOI: https://doi.org/10.1038/ S41598-018-20462-6

[34] S. Mondal and P. Bours. 2017. Person identification by keystroke dynamics using pairwise user coupling. IEEE Trans. Inf. Forens. Secur. 12, 6 (June 2017), 1319-1329. DOI : https://doi.org/10.1109/TIFS.2017.2658539

[35] Soumik Mondal and Patrick Bours. 2017. A study on continuous authentication using a combination of keystroke and mouse biometrics. Neurocomputing 230 (2017), 1-22. DOI : https://doi.org/10.1016/j.neucom.2016.11.031

[36] Fabian Monrose and Aviel Rubin. 1997. Authentication via keystroke dynamics. In Proceedings of the 4th ACM Conference on Computer and Communications Security (CCS'97). ACM, New York, NY, 48-56. DOI : https://doi.org/10.1145/ 266420.266434

[37] Tuan Nguyen and Jonathan Voris. 2017. Touchscreen biometrics across multiple devices. In Proceedings of the USENIX Symposium on Usable Privacy and Security (SOUPS'17).

[38] M. Obaidat and Balqies Sadoun. 1997. Verification of computer users using keystroke dynamics. IEEE Trans. Syst. Man Cyber. Part B: Cyber. 27, 2 (1997)

[39] Avar Pentel. 2019. Predicting user age by keystroke dynamics. In Artificial Intelligence and Algorithms in Intelligent Systems, Radek Silhavy (Ed.). Springer International Publishing, Cham, 336-343.

[40] Paulo Henrique Pisani and Ana Carolina Lorena. 2013. A systematic review on keystroke dynamics. f. Braz. Comput. Soc. 19, 4 (1 Nov. 2013), 573-587. DOI : https://doi.org/10.1007/s13173-013-0117-7

[41] Oxford University Press. 2011. The Oxford English corpus: Facts about the language. OxfordDictionaries.com. Retrieved from https://web.archive.org/web/20111226085859/ Retrieved from http://oxforddictionaries.com/words/ the-oec-facts-about-the-language. 
[42] K. A. Rahman, K. S. Balagani, and V. V. Phoha. 2013. Snoop-forge-replay attacks on continuous verification with keystrokes. IEEE Trans. Inf. Forens. Sec. 8, 3 (Mar. 2013), 528-541. DOI : https://doi.org/10.1109/TIFS.2013.2244091

[43] Kenneth Revett, Sérgio Tenreiro de Magalhães, and Henrique M. D. Santos. 2005. Enhancing login security through the use of keystroke input dynamics. In Advances in Biometrics, David Zhang and Anil K. Jain (Eds.). Springer Berlin, 661-667.

[44] Seong seob Hwang, Sungzoon Cho, and Sunghoon Park. 2009. Keystroke dynamics-based authentication for mobile devices. Comput. Sec. 28, 1 (2009), 85-93. DOI: https://doi.org/10.1016/j.cose.2008.10.002

[45] Abdul Serwadda and Vir V. Phoha. 2013. Examining a large keystroke biometrics dataset for statistical-attack openings. ACM Trans. Inf. Syst. Sec. 16, 2, Article 8 (Sept. 2013), 30 pages. DOI : https://doi.org/10.1145/2516960

[46] Yong Sheng, Vir Phoha, and S. M. Rovnyak. 2005. A parallel decision tree-based method for user authentication based on keystroke patterns. IEEE Trans. Syst. Man Cyber. Part B: Cyber. 35 (9 2005), 826-833. DOI : https://doi.org/10.1109/ TSMCB.2005.846648

[47] D. Shukla and V. V. Phoha. 2019. Stealing passwords by observing hands movement. IEEE Trans. Inf. Forens. Sec. 14, 12 (Dec. 2019), 3086-3101. DOI : https://doi.org/10.1109/TIFS.2019.2911171

[48] T. Sim and R. Janakiraman. 2007. Are digraphs good for free-text keystroke dynamics? In Proceedings of the IEEE Conference on Computer Vision and Pattern Recognition. 1-6. DOI : https://doi.org/10.1109/CVPR.2007.383393

[49] D. Stefan and D. Yao. 2010. Keystroke-dynamics authentication against synthetic forgeries. In Proceedings of the 6th International Conference on Collaborative Computing: Networking, Applications and Worksharing (CollaborateCom'10). 1-8. DOI : https://doi.org/10.4108/icst.collaboratecom.2010.16

[50] A. E. Sulavko, A. V. Eremenko, and A. A. Fedotov. 2017. Users' identification through keystroke dynamics based on vibration parameters and keyboard pressure. In Proceedings of the Dynamics of Systems, Mechanisms and Machines Conference (Dynamics'17). 1-7. DOI : https://doi.org/10.1109/Dynamics.2017.8239514

[51] A. Sulong, Wahyudi, and M. U. Siddiqi. 2009. Intelligent keystroke pressure-based typing biometrics authentication system using radial basis function network. In Proceedings of the 5th International Colloquium on Signal Processing and Its Applications. 151-155. DOI : https://doi.org/10.1109/CSPA.2009.5069206

[52] Yan Sun, H. Ceker, and S. Upadhyaya. 2017. Anatomy of secondary features in keystroke dynamics -achieving more with less. In Proceedings of the IEEE International Conference on Identity, Security and Behavior Analysis (ISBA'17). 1-6. DOI : https://doi.org/10.1109/ISBA.2017.7947691

[53] Pin Shen Teh, Andrew Beng Jin Teoh, and Shigang Yue. 2013. A survey of keystroke dynamics biometrics. Sci. World f. (Sept. 2013). DOI : https://doi.org/10.1155/2013/408280

[54] Ioannis Tsimperidis, Avi Arampatzis, and Alexandros Karakos. 2018. Keystroke dynamics features for gender recognition. Dig. Investig. 24 (2018), 4-10. DOI : https://doi.org/10.1016/j.diin.2018.01.018

[55] Mindaugas Ulinskas, Robertas Damaševičius, Rytis Maskeliūnas, and Marcin Woźniak. 2018. Recognition of human daytime fatigue using keystroke data. Procedia Comput. Sci. 130 (2018), 947-952. DOI : https://doi.org/10.1016/j.procs. 2018.04.094

[56] Umphress and G. Williams. 1985. Identity verification through keyboard characteristics. Int. F. Man-Mach. Stud. 23, 3 (1985), 263-273.

[57] Changsheng Wu, Wenbo Ding, Ruiyuan Liu, Jiyu Wang, Aurelia C. Wang, Jie Wang, Shengming Li, Yunlong Zi, and Zhong Lin Wang. 2018. Keystroke dynamics enabled authentication and identification using triboelectric nanogenerator array. Mater. Today 21, 3 (2018), 216-222. DOI : https://doi.org/10.1016/j.mattod.2018.01.006

[58] Tong Zhu, Qiang Ma, Shanfeng Zhang, and Yunhao Liu. 2014. Context-free attacks using keyboard acoustic emanations. In Proceedings of the ACM SIGSAC Conference on Computer and Communications Security (CCS'14). ACM, New York, NY, 453-464. DOI : https://doi.org/10.1145/2660267.2660296

[59] John Zulueta, Piscitello Andrea, Mladen Rasic, Rebecca Easter, Pallavi Babu, Scott Langenecker, Melvin McInnis, Olusola Ajilore, Peter Nelson, Kelly Ryan, and Alex Leow. 2017. Predicting mood disturbance severity in bipolar subjects with mobile phone keystroke dynamics and metadata. Bio. Psych. 81, 10 (May 2017).

Received July 2018; revised September 2019; accepted December 2019 